\title{
Structure of subtidal algal assemblages on soft- bottom sediments: fauna/flora interactions and role of disburbances in the Bay of Brest, France
}

\author{
Christian Hily ${ }^{1, *}$, Philippe Potin ${ }^{1}$, Jean-Yves Floc' ${ }^{2}$ \\ ${ }^{1}$ CNRS, LP 4601, Station de Biologie marine, F-29211 Roscoff, France \\ ${ }^{2}$ Laboratoỉre de Physiologie végétale, Institut d'Etudes Marines, Université de Bretagne Occidentale, Avenue Le Gorgeu,
} F-29287 Brest Cédex, France

\begin{abstract}
Algal and faunal assemblages living on soft bottom sediments were studied in the Bay of Brest, France, using quantitative descriptions of the distribution, diversity and biomass of epibenthic species. Similar classification and ordination analyses were used on floral and animal data. Dense assemblages of macrophytes, mainly Rhodophyceae, were found to grow on sandy mud sediments. Sediments were essentially covered by maerl beds of the calcareous red alga Lithothamnion corallioides and various shell substrates. Five main algal assemblages extended along a west-east decreasing gradient of hydrodynamic activity (i.e. tidal currents, waves and turbidity). The high frequency of storms during the year is the main feature which disturbs both flora and epifauna. Interactions between flora and fauna were either direct, e.g. grazing and spatial competition, or indirect, most of the substrata occupied by macrophytes being shells of dead bivalves and gastropods. Moreover attachment of algae was largely dependent on population dynamics of shell species living in the area. As a consequence, in the most disturbed area, the macrophytes found were opportunistic species (Polysiphonia fibrillosa and Polysiphonia urceolata). In areas where the assemblage was unstructured, a fact which prevented the development of the herbivorous species, the animal assemblage was then dominated by suspension feeders (Ficulina ficus and Phallusia mammillata) which competed for space with the macrophytes. In the least disturbed area, the herbivorous species dominated the fauna assemblage while flora assemblage had a high level of organization. However, in those places eutrophication and high turbidity prevented the establishment of many algal species, causing the ubiquitous ones to dominate (Ulva sp., Ceramium rubrum). Between these extreme areas, the living Lithothamnion beds were more developed and were defined as a characteristic assemblage of diversified Rhodophyceae species such as Chylocladia verticillata, Halarachnion ligulatum, Crytopleura ramosa. Finally, the role of wave disturbance as the main factor controlling algal assemblages of shallow soft-bottom sediments is discussed.
\end{abstract}

\section{INTRODUCTION}

In temperate latitudes, subtidal unstable substrates and soft bottoms of shallow waters are generally colonized by erect species of seaweeds during a short growing season, but crustose algae are well known to be abundant throughout the year. Several authors described the algal vegetation as growing on shell bottoms (Kjellman 1878, Waern 1958, Sears \& Wilce 1975),

\footnotetext{
- Present address: Centre ORSTOM, BP 529 Papeete, Tahiti. French Polynesia
}

cobble habitats (Liebermann et al. 1979, Davis \& Wilce 1987a) or maerl beds (Cabioch 1969, Blunden et al. 1977, 1981). The ecology of these communities, however, had received little examination, in contrast to the abundant literature about kelp forest (Ojeda \& Dearborn 1989, Underwood \& Kennelly 1990).

Two accounts were made of the ecology of subtidal algae on cobble substrates taken off the west coast of Ghana (Lieberman et al. 1979) and in Plum Cove, Massachusetts, USA (Davis \& Wilce 1987b). Sears \& Wilce (1975) studied the associations and the seasonal variation of flora in an unstable shell bottom habitat off 
Cape Cod. The ecology of loose-lying coralline algae (maerl) and associated epiflora has not been investigated since the descriptive work of Cabioch (1969) in the Bay of Morlaix, France.

The main ecological mechanism responsible for the control of the community structure in those habitats was recognized as a physical disturbance (Davis \& Wilce 1987b). Disturbance is defined by Grime (1977) as an 'event, biotic or abiotic in origin, that destroys biomass, affecting from one organism to entire communities'. Subtidal physical disturbance tends to be seasonal, but it is difficult to measure the influence that storms have on the substrata. During a quantitative algal study of a soft bottom from the Bay of Brest, France, we used classification and ordination techniques to elucidate the spatial patterns of algal and faunal assemblages and the factors controlling community structure. The interest of simultaneous algal and epifaunal investigations in determining the major factors and their global influence on the communities' organization is discussed.

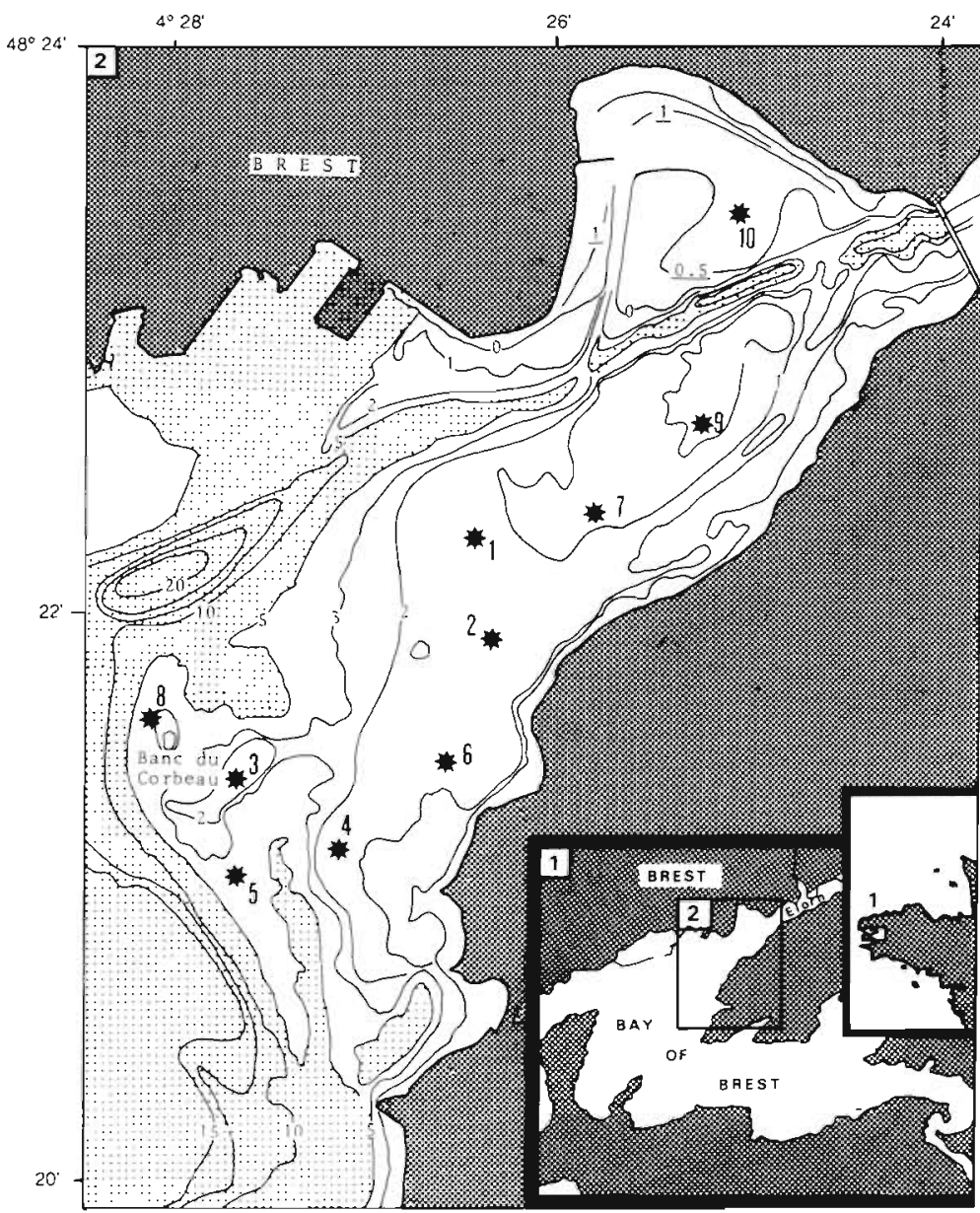

Fig. 1. Sampling stations and bathymetry for study area, Bay of Brest, France

\section{MATERIALS AND METHODS}

Study site. Research was conducted in the northern part of the Bay of Brest $\left(48^{\circ} 24^{\prime} 00^{\prime \prime} \mathrm{N}, 04^{\circ} 33^{\prime} 00^{\prime \prime} \mathrm{W}\right.$ to $48^{\circ} 16^{\prime} 50^{\prime \prime} \mathrm{N}^{0} 04^{\circ} 1500^{\prime \prime} \mathrm{W}$ ), western Brttany, France. The study site was situated between the sheltered estuarine waters (eastern part) and the open marine waters (western part); salinities varied from 30 to $34 \%$. The bottom consisted of a shallow muddy sediment ( 0 to $5 \mathrm{~m}$ depth) covered by shells and Lithothamnion corallioides Crouan fragments, which are favorable substrata allowing the fixation and growth of macrophytes and epifauna (Hily 1989, 1991). Water circulation induced by tidal currents is alternating and residual transport of particles is directed to the open sea (Salomon 1980). Those tidal currents are not strong enough to erode the sediment surface (current speed $<1$ knot on spring tides). It is a natural sedimentation zone (Fichaut 1984), but the frequent storms can induce a high resuspension of detritic material and maintain a very high turbidity $\left(>100 \mathrm{mg} \mathrm{l}^{-1}\right)$ over long periods of time. The swell from the open sea breaks on the western part of the banks (Banc du Corbeau) and consequently prevents sedimentation in this area (Fig. 1).

The effluents of Brest (250000 inhabitants) on the northern coast of the site, and residues from intensive agricultural activity on the drainage basin of the Elorn River $\left(400 \mathrm{~km}^{2}\right)$, induce a heavy discharge of organic effluents and higin levels of nutrients $\left(1000 \mu \mathrm{g}\right.$-at. $\mathrm{NO}_{3}^{-} 1^{-1}$ in the fall and winter) in waters of the studied area (Treguer \& Queguiner 1989).

Sampling. Between spring 1987 and spring 1988, 2 different investigations were performed. First, an underwater video camera (OSPREY S.I.T.) hung on a support was suspended just above the substratum and periodically laid on the floor of the bay (observed surface $=400$ $\mathrm{cm}^{2}$ ) to observe sediment surface characteristics, and quantify the algal cover and larger epifauna. Each station was a transect $200 \mathrm{~m}$ long. A total of 65 stations were sampled in the investigated area during 3 surveys (June 1987, March 1988, June 1988). Second, quantitative samples were collected by scuba divers. Each sample consisted of the total epiflora and epifauna collected within a $1 \mathrm{~m}^{2}$ metallic frame placed on the sediment surface by divers. Four to six samples were collected at each of 10 stations (Fig. 1). After identification, macroalgae were dried, then 
weighed to the nearest $0.1 \mathrm{~g}$; the epifauna were numbered and weighed $\left(\mathrm{g} \mathrm{m}^{-2}\right.$, organic dry weight).

Data analysis. Characteristics of the algal and faunal distribution in the study area were investigated in terms of number of species, number of individuals, and biomass. Community diversity as defined by Shannon's equation $\left(H^{\prime}=P \log P\right.$ ) was analyzed. Rank-frequency diagrams were used to interpret the structural organisation of assemblages. The rank frequencies diagrams give a better expression of the community structure than the diversity index ( $H^{\prime}$ shannon) or equitability. In such diagrams, species are ranked by decreasing order of abundance: for a species $A$ the log of dominance is plotted against the log rank $A$ (Frontier 1976, Hily 1983).

In order to search for patterns among the biological variables, data were arranged in a matrix in which the $N$ samples are described by $S$ species. For algae, data were expressed in terms of biomass and for fauna, in terms of density. After logarithmic transformation of the data, the measurement of similarity adopted was the $x^{2}$ distance (Benzecri 1973) as recommended by Legendre \& Legendre (1979). From the similarity matrix the group-average hierarchical sorting strategy was retained to distinguish groups of samples and species. The next stage of analysis was the multivariate analysis (correspondence factorial analysis; Lebart et al. 1982) which can situate each sample and species in relation to all the others and can then temper the discontinuities between the observations often emphasized by the method of classification (Field et al 1982).

Table 1. Sediment surface characteristics in the different units of the study area. Litho.: Lithothamnium; SOM: soft mud; HES: heterogenous sand; DLL: dense living Lithothamnium; MIL: mixed Lithothamnium; DEL: dead Lithothamnium; SHM: shelly mud

\begin{tabular}{|lcccc|}
\hline & $\begin{array}{c}\text { Pelitic } \\
\text { fraction } \\
<63 \mu \mathrm{m}(\%)\end{array}$ & $\begin{array}{c}\text { Living } \\
\text { Litho. } \\
(\%)\end{array}$ & $\begin{array}{c}\text { Dead } \\
\text { Litho. } \\
(\%)\end{array}$ & Shells \\
\hline SOM & $60-90$ & 0 & $0-20$ & $0-40$ \\
HES & $5-30$ & 0 & $0-20$ & $10-60$ \\
DLL & $30-80$ & $80-100$ & $0-40$ & $0-10$ \\
MIL & $30-80$ & $20-80$ & $20-80$ & $10-40$ \\
DEL & $30-80$ & $0-20$ & $80-100$ & $0-20$ \\
SHM & $30-80$ & 0 & $0-20$ & $60-100$ \\
\hline
\end{tabular}

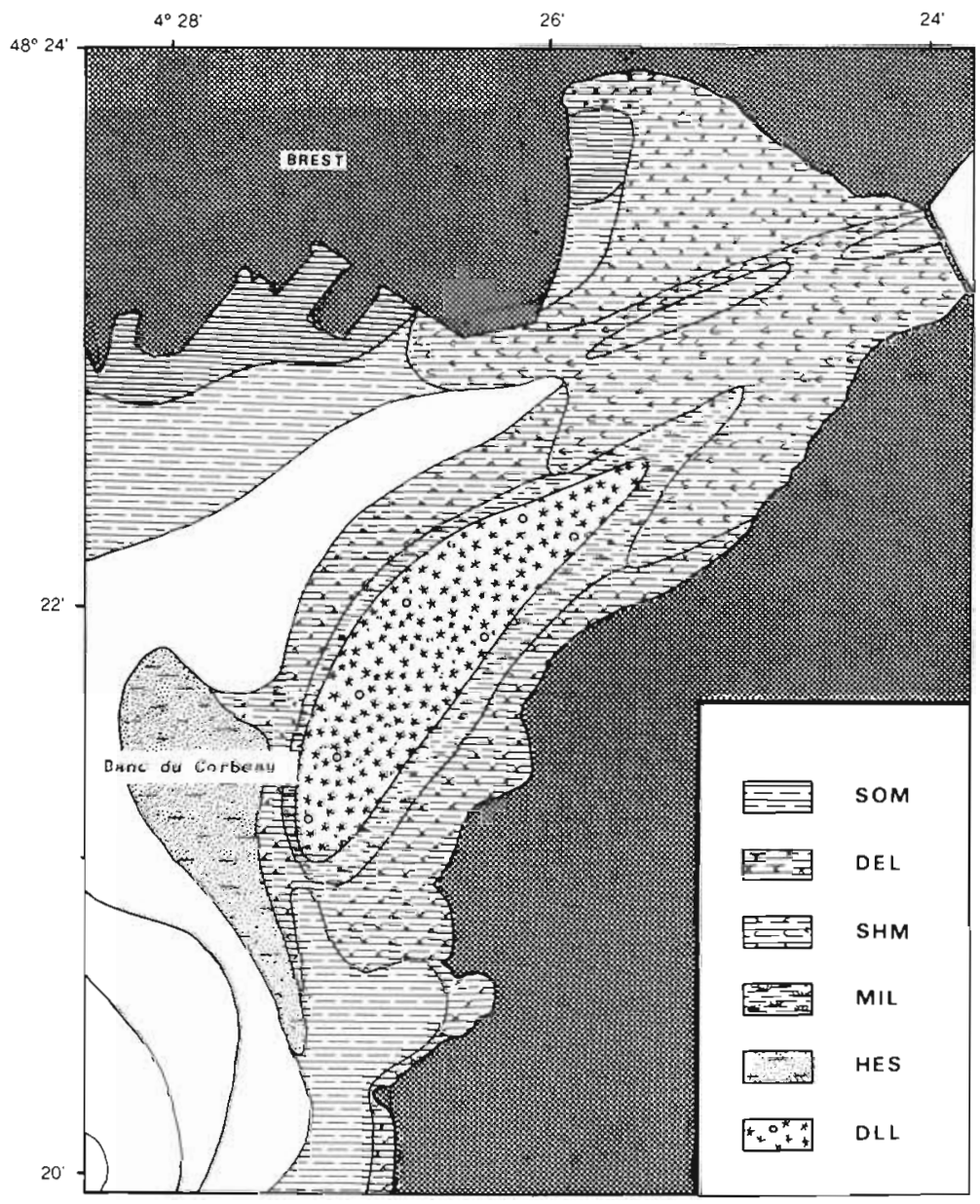

Fig. 2. Bottom sediment characteristics in the study area. SOM: soft mud; DEL: dead Lithothamnion; SHM: shelly heterogenous mud; MIL: mixed Lithothamnion; HES: heterogenous sand; DLL: dense living Lithothamnion

\section{RESULTS}

\section{Habitat description}

Substratum quality is an important factor in the explanation of the spatial distribution of algal and faunal assemblages. To determine the structure of the sediment surface, numerous transects within the study area were observed in the winter with the underwater video camera. During this period the algal cover had almost totally disappeared and substrata could be observed. Six types were defined on these shallow muddy banks in relation to several descriptors of the bottom structure: pelitic fraction, fraction of living and dead Lithothamnion on the sediment surface and abundance of shells and cobbles (Table 1, Fig. 2):

Soft mud (SOM): Sediment surface is free from shells, cobbles and Lithothamnion fragments, which prevent the settlement of algae. 
Heterogenous sand (HES): On the exposed area sediment is coarse sand with cobbles and gravel (Banc du Corbeau).

Dense living Lithothamnion (DLL): The central part of the study area is colonized by a dense $(3$ to $5 \mathrm{~cm}$ deep) living Lithothamnion corallioides population which entirely covers the bottom.

Dead Lithothamnion (DEL): Around this DDL area, sediment is sandy mud with numerous macrofragments of Lithothamnion carried by waves from the DLL and MIL unit. It is a high sedimentation zone of the terrigenous material carried by the Elorn River.

Mixed Lithothamnion (MIL): This is a transition between DLL and DEL units.

Shelly mud (SHM): Sediment surface structure is the result of the accumulation of numerous shells of bivalves (e.g. Venerupis sp., Ostrea edulis Linnaeus, Lutraria lutraria Linnaeus) and gastropods (Crepidula fornicata Linnaeus) which can totally cover the bottom.

\section{Algal assemblages}

\section{Community structure}

Seventy-seven taxa were identified from the samples collected in June 1987 and June 1988. The dry biomass of 58 species, other than most calcareous or crustose red and brown algae, were recorded and are listed in Table 2. Rhodophyta was the most abundant component of the flora. Only one loose-lying coralline species composed the maerl beds; Lithothamnium corallioides (var. corallioides Cabioch, 1966) is the most abundant species of seaweed in the study area and mainly contributed to the carbonate equilibrium by calcium carbonate secretion (Potin et al. 1990). In our study, however, the maerl is considered as a substrate for attached algae rather than as an alga. The Rhodophyta Polysiphonia fibrillosa Dillwyn (same as Polysiphonia violacea Hudson (Parke \& Dixon 1976) was omnipresent and contributed to the highest biomass.

Large variations of the diversity index occurred between the sampling stations. Low values were obtained at Stns 7 and $5\left[H^{\prime}(\mathrm{SE}): 0.75(0.3)\right.$ and $1.51(0.5)$ respectively]. Mean values $\left(2<H^{\prime}<3\right.$ ) were obtained at Stns $2,10,9,6$ and $8(2.4,2.41,2.48,2.85$ and 2.9 respectively). Three stations obtained $H^{\prime}$ values $>3$ (Stns 4,1 and 3: 3.25, 3.28 and 3.38 respectively). Stn 2 was characterized by the high variability of the diversity in the different samples (SE $=0.91$ ).

Comparison of rank frequency diagrams for the 10 stations allowed them to be placed in 1 of 3 groups based on curve shape (Fig. 3). Stns 1, 2, 3, 5 and 7 had straight curves which characterized low structured communities with 1 or 2 species largely dominant. The total number of species was low but biomass was high; Polysiphonia fibrillosa was the dominant species for this group of stations. Stns 4, 6, 8 and 10 had curved diagrams which characterized structured communities where ca 10 species had frequencies higher than $10 \%$. Stn 9 was the only station in which the high concavity of the diagram gave the expression of a mature community.

\section{Classification}

$\chi^{2}$ distance similarity coefficients were calculated between samples. Dendrograms produced by groupaverage clustering of these values showed 5 distinct clusters at approximately $55 \%$ similarity and 4 single samples. These clusters consisted of samples from different stations. Clusters A and B subdivided respectively into 2 and 3 clusters at ca $75 \%$ similarity (Fig. 4). From the habitat characteristics described before, the clusters were interpreted as: (A) heterogenous assemblage, sandy mud with boulders; (B1 and B2) living maerl; (B3) dead maerl; (C) Ficulina ficus Olivi assemblage, heterogeneous sand with mud; (D) opportunistic algae assemblage, heterogeneous sand with mud and cobbles, highest biomass of Polysiphonia fibrillosa;

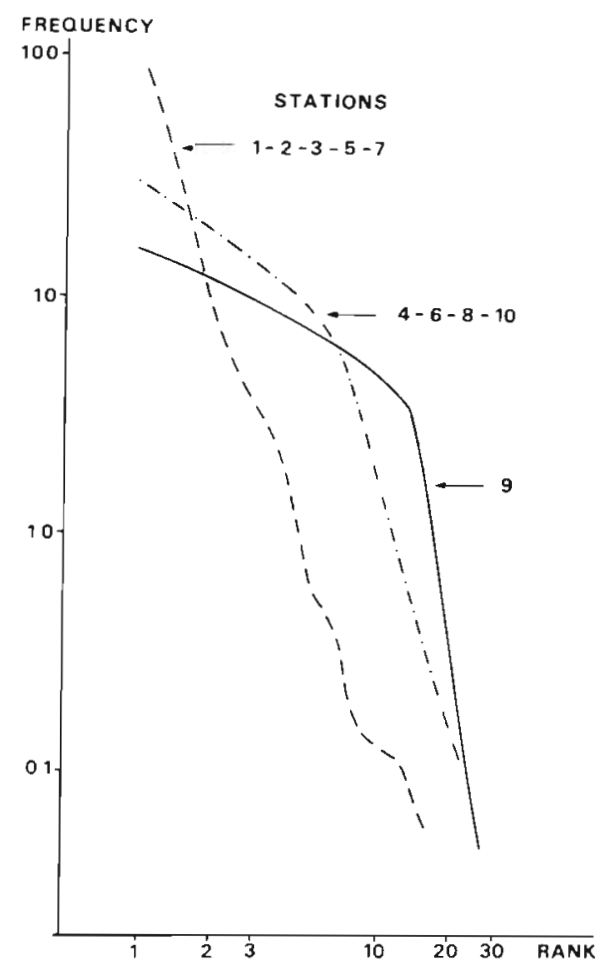

Fig. 3. Rank frequency diagrams: (- - ) average curve of Stns 1, 3, 5 and 7; (-.-..-.) average curve of Stns 4,6,8 and $10_{i}(-)$ curve of $\operatorname{Stn} 9$. Rank of species was obtained from the relative frequency (= dominance) in $\%$ 


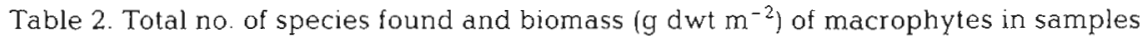

\begin{tabular}{|c|c|c|c|c|c|c|c|c|c|c|c|c|c|}
\hline Species & \multirow[t]{2}{*}{ Code } & \multicolumn{6}{|c|}{$\operatorname{Stn} 1$} & \multicolumn{6}{|c|}{$\sin 2$} \\
\hline Sample no: & & 11 & 12 & 13 & 14 & 15 & 16 & 21 & 22 & 23 & 24 & 25 & 26 \\
\hline \multicolumn{14}{|l|}{ Chlorophyceae } \\
\hline Cladophora pellucida & $\mathrm{clp}$ & - & - & - & - & - & - & - & - & - & - & - & - \\
\hline Cladophorasp. & cla & - & - & - & - & - & - & - & - & - & - & - & - \\
\hline Enteromorphasp. & ent & - & - & - & - & - & - & - & - & - & - & 0.02 & - \\
\hline Ulva sp. & ulv & 0.01 & 0.17 & 0.01 & - & 0.02 & 0.05 & - & - & - & - & 0.13 & 0.5 \\
\hline \multicolumn{14}{|l|}{ Pheophyceae } \\
\hline Asperococcus sp. & asp & - & - & - & - & - & - & - & - & - & - & - & - \\
\hline Colpomenia peregrina & cop & - & - & - & - & - & - & - & - & - & - & - & -- \\
\hline Cutleria multifida & cum & - & 0.01 & 0.01 & 0.01 & 0.01 & 0.02 & - & 0.01 & 0.01 & - & - & - \\
\hline Desmarestia ligulata & del & - & - & - & - & - & - & - & - & - & - & - & - \\
\hline Dictyota dichotoma & did & 0.25 & 0.17 & 0.17 & 0.10 & 0.01 & 0.82 & 1.29 & 1.38 & 0.04 & 2.26 & 8.82 & 7.01 \\
\hline Ectocarpalese & ect & - & - & - & - & - & - & - & - & - & - & - & - \\
\hline Laminaria sacchanna & las & - & - & - & - & - & - & - & - & 1.15 & 0.60 & - & - \\
\hline Punctaria plantaginea & pup & - & - & - & - & - & - & - & - & - & - & - & - \\
\hline Sacchoriza polyschides & sac & - & - & - & - & - & - & - & - & 0.96 & - & - & - \\
\hline Pheophyceae indeterminate & phi & - & - & - & - & - & - & - & - & - & - & - & - \\
\hline \multicolumn{14}{|l|}{ Rhodophyceae } \\
\hline Antithamnion plumula & anp & - & - & - & & - & _- & - & - & - & - & - & - \\
\hline Apoglossum ruscifolium & apr & - & - & - & - & - & - & - & - & - & - & - & -- \\
\hline Brongniartella byssoides & brb & 0.15 & 0.05 & 0.08 & 0.06 & 0.03 & 0.03 & - & 0.07 & - & 0.03 & - & 0.50 \\
\hline Calliblepharis ciliata & $\mathrm{cac}$ & - & - & - & - & - & - & - & - & 0.09 & - & - & 0.02 \\
\hline Calliblepharis jubata & $\mathrm{caj}$ & - & 0.01 & - & - & - & - & 0.21 & 0.17 & - & - & 0.03 & 0.03 \\
\hline Callophyllis flabellata & caf & 0.04 & 0.01 & - & 0.01 & 0.01 & - & 2.65 & 0.02 & 0.35 & 0.50 & 2.55 & - \\
\hline Ceramium echionotum & cee & 0.56 & 0.09 & 0.12 & - & - & 0.12 & - & - & - & 0.01 & 0.21 & 0.03 \\
\hline Ceramium rubrum & cer & - & - & - & - & 0.02 & 0.01 & - & - & - & - & - & - \\
\hline Champia parvula & $\operatorname{chp}$ & 0.06 & 0.01 & 0.01 & - & - & 0.33 & 0.04 & - & - & - & 0.17 & 0.01 \\
\hline Chondria dasyphylla & chd & 0.94 & 0.12 & 0.33 & 0.03 & 0.30 & 0.33 & 0.05 & 0.01 & - & 0.52 & 1.65 & 0.62 \\
\hline Chylocladia verticillata & chv & - & 0.41 & 0.03 & - & 0.04 & 0.01 & 5.45 & 0.19 & 0.10 & - & 0.17 & - \\
\hline Cordylecladia erecta & coe & 0.21 & 0.08 & 0.04 & 0.01 & 0.02 & 0.01 & - & - & - & - & - & 0.02 \\
\hline Cryptopleura ramosa & $\mathrm{crr}$ & - & - & 0.11 & - & - & - & 4.56 & 3.67 & - & 2.15 & 7.06 & 2.53 \\
\hline Dudresnaya verticillata & duv & - & - & - & - & - & - & - & - & - & - & - & - \\
\hline Gracilaria bursa-pastons & grb & - & - & - & - & - & - & - & - & - & - & 0.05 & - \\
\hline Gracilaria folufera & grf & 0.05 & 0.04 & 0.04 & - & 0.06 & 0.02 & 0.03 & - & - & 0.08 & 0.04 & 0.04 \\
\hline Gracilana verrucosa & grv & 0.09 & 0.07 & 0.09 & 0.03 & 0.11 & 0.04 & 0.05 & - & - & 0.22 & 0.40 & 0.07 \\
\hline Gymnogongrus crenulatus & gyc & - & 0.21 & - & - & - & - & - & - & - & - & - & - \\
\hline Gymnogongrus griffithsiae & gyg & - & 0.05 & 0.02 & - & 0.01 & 0.01 & - & - & - & - & - & - \\
\hline Halarachnion hgulatum & hal & 0.15 & 0.01 & 0.03 & - & 0.05 & 0.02 & 0.30 & 0.16 & - & 0.44 & 3.17 & 2.28 \\
\hline Halymenia latifolia & hay & 0.04 & 0.04 & - & - & - & 0.01 & - & - & - & - & 0.01 & - \\
\hline Heterosiphonia plumosa & hep & 0.04 & - & 0.01 & _- & - & 0.01 & - & - & - & - & - & 0.03 \\
\hline Hypoglossum hypoglossoides & hyh & 0.14 & 0.03 & 0.24 & 0.18 & 0.02 & 1.97 & 0.89 & 0.21 & - & 0.44 & 1.33 & - \\
\hline Kallymenia reniformis & kar & 0.02 & 0.51 & 0.04 & - & - & - & 0.49 & 0.69 & - & - & - & 1.08 \\
\hline Microcladia glandulosa & mig & 0.13 & - & 0.06 & 0.36 & 0.07 & 0.29 & 0.37 & 0.47 & - & 0.03 & 0.36 & 0.05 \\
\hline Nitophyllum punctatum & nip & - & - & 0.05 & 0.01 & - & - & 9.57 & 0.97 & - & 0.83 & 5.88 & 4.07 \\
\hline Phyllophora crispa & phe & - & - & - & - & - & - & - & - & - & - & - & - \\
\hline Phyllophora pseudoceranordes & php & - & - & - & - & - & - & - & - & - & - & - & - \\
\hline Plocamium cartilagineum & $\mathrm{plc}$ & - & - & 0.04 & - & - & 0.01 & - & - & 0.08 & 0.02 & 0.03 & - \\
\hline Polyneura hilliae & poh & - & - & 0.03 & - & - & 0.01 & - & - & - & - & - & - \\
\hline Polysiphonia elongata & poe & 0.06 & 0.04 & 0.05 & 0.16 & 0.06 & - & - & - & - & 0.03 & 0.03 & - \\
\hline Polysiphona fibrillosa & pof & 9.28 & 15.84 & 18.60 & 19.51 & 12.57 & 7.54 & 8.39 & 3.64 & 52.00 & 5.70 & 3.04 & 6.93 \\
\hline Polysiphonia macrocarpa & pom & - & - & - & - & - & - & - & - & - & - & - & - \\
\hline Polysiphonia nigra & pon & - & - & - & - & - & 0.02 & - & - & - & - & - & - \\
\hline Polysiphonia urceolata & pou & 0.56 & 1.63 & 1.69 & - & 0.87 & 2.62 & - & 0.06 & 2.31 & 0.74 & 1.68 & 0.05 \\
\hline Pterosiphonia parasitica & pta & - & 0.02 & - & - & 0.01 & 0.01 & - & - & - & - & - & - \\
\hline Pterosiphonia pennata & pte & - & - & 0.02 & - & - & 0.01 & - & - & - & 0.01 & 0.01 & 0.01 \\
\hline Rhodophyllis divaricata & rhd & 0.15 & - & - & 0.03 & - & - & 0.16 & - & - & 0.20 & - & 0.03 \\
\hline Rytiphlaea tinctoria & Tyt & - & - & - & - & - & - & - & & - & - & - & - \\
\hline Solieria chordalis & soc & 0.04 & 0.04 & - & 0.01 & - & 0.01 & - & - & 0.01 & - & - & 0.01 \\
\hline Sphaerococcus coronopifolius & $\mathrm{spc}$ & - & - & - & - & - & - & - & - & - & - & - & - \\
\hline Sphondylothamnon multifidum & $\mathrm{spm}$ & - & - & _- & - & - & - & - & - & - & - & - & - \\
\hline Spyridia filamentosa & spf & 0.19 & 0.03 & 0.07 & 0.01 & 0.21 & 0.10 & 0.17 & 0.29 & 0.23 & - & 0.23 & 0.24 \\
\hline Stenogramme intersupta & sti & 0.32 & 0.08 & 0.04 & - & 0.12 & 0.41 & 0.14 & 0.25 & 0.10 & 0.03 & 0.25 & 0.05 \\
\hline$N$ (total no. of species) & & 23 & 26 & 27 & 15 & 21 & 28 & 18 & 17 & 12 & 21 & 25 & 24 \\
\hline B (total biomass, $\mathrm{g}$ ) & & 13.45 & 19.74 & 22.03 & 20.52 & 14.62 & 14.84 & 34.81 & 13.26 & 57.30 & 15.07 & 37.03 & 26.05 \\
\hline $\mathrm{SB}$ (mean biomass per station, $\mathrm{g}$ ) & & & & 17.5 & & & & & & 30.5 & & & \\
\hline
\end{tabular}


Table 2 (continued)

\begin{tabular}{|c|c|c|c|c|c|c|c|c|c|c|c|c|c|c|c|c|c|}
\hline \multirow{2}{*}{$\begin{array}{l}\text { Species } \\
\text { code }\end{array}$} & \multicolumn{3}{|c|}{$\sin 3$} & \multicolumn{3}{|c|}{$\operatorname{Stn} 4$} & \multicolumn{6}{|c|}{$\operatorname{Stn} 5$} & \multicolumn{5}{|c|}{$\operatorname{Sin} 6$} \\
\hline & 31 & 32 & 33 & 41 & 42 & 43 & 51 & 52 & 53 & 54 & 55 & 56 & 61 & 62 & 63 & 64 & 65 \\
\hline clp & - & - & - & - & - & - & - & - & - & - & - & - & - & - & - & - & - \\
\hline cla & - & - & - & - & - & - & - & - & - & - & - & - & - & - & - & - & - \\
\hline ent & - & - & - & - & - & - & - & - & - & - & - & - & - & - & - & - & - \\
\hline ulv & 0.23 & 0.42 & 12.04 & 0.06 & 0.24 & 0.21 & - & - & - & - & - & - & - & 0.01 & 0.30 & 0.11 & 0.27 \\
\hline asp & - & - & - & - & - & - & - & - & - & - & - & - & - & - & - & - & - \\
\hline cop & - & - & - & -. & - & - & - & - & - & - & - & - & - & - & - & - & - \\
\hline cum & 0.01 & 0.01 & - & - & - & - & - & - & - & - & - & - & - & - & - & - & - \\
\hline del & - & - & - & - & - & - & - & - & - & - & - & - & - & - & - & - & - \\
\hline did & 0.95 & 0.21 & 0.06 & 2.04 & 1.19 & 4.99 & - & - & - & - & - & - & 1.00 & 0.53 & 0.16 & 1.57 & 1.49 \\
\hline ect & - & - & - & - & - & - & - & - & - & - & - & - & - & - & - & - & - \\
\hline las & 0.14 & 0.70 & 3.01 & - & 0.45 & 0.98 & - & - & - & 0.27 & 1.04 & 0.05 & - & - & - & - & - \\
\hline pup & - & - & - & - & - & - & - & - & - & - & - & - & - & - & - & - & - \\
\hline sac & - & - & 1.10 & - & - & - & - & - & - & - & - & - & - & - & - & - & - \\
\hline phi & - & - & - & - & - & - & - & - & - & - & - & - & - & - & - & - & - \\
\hline anp & - & - & - & - & - & - & - & - & - & - & - & - & - & - & - & - & - \\
\hline apr & - & - & - & - & - & - & - & - & - & - & - & - & - & - & - & - & 0.56 \\
\hline brb & 1.09 & 0,12 & 0.23 & 0.11 & 0.15 & 0.44 & 0.20 & 0.23 & 0.47 & 0.73 & 1.38 & 0.41 & 0.47 & 1.17 & 0.39 & 2.64 & 3.10 \\
\hline cac & 0.04 & 0.13 & - & - & - & - & - & - & - & - & - & - & & - & - & - & - \\
\hline caj & - & - & - & 0.21 & - & 0.41 & - & - & - & - & 0.10 & - & 0.04 & - & - & - & 0.02 \\
\hline caf & 0.37 & 0.10 & 0.21 & - & - & - & - & - & 0.90 & - & - & - & 0.03 & - & - & 0.05 & - \\
\hline cee & - & - & - & - & - & - & - & - & - & - & 0.03 & 0.20 & 0.32 & 0.35 & 0.86 & 2.74 & 2.45 \\
\hline cer & - & - & - & - & 0.10 & 0.11 & - & - & - & - & - & - & - & 0.02 & 0.04 & - & - \\
\hline chp & - & - & - & - & 0.05 & - & - & - & - & - & - & - & - & - & - & - & 1.27 \\
\hline chd & 0.04 & 0.13 & - & - & - & - & - & 0.04 & - & - & - & - & - & 0.38 & 0.24 & 1.01 & 1.01 \\
\hline chv & - & - & - & 4.78 & - & 3.96 & - & - & - & - & - & - & - & 0.01 & 0.02 & - & 0.19 \\
\hline coe & 0.02 & 0.05 & _- & - & - & - & - & - & - & - & - & - & - & - & - & - & - \\
\hline CrI & - & 0.65 & 0.63 & 3.25 & 4.04 & 2.31 & - & - & - & - & - & - & 10.82 & 1.51 & 2.87 & 5.41 & 5.64 \\
\hline duv & 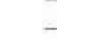 & - & - & - & - & - & - & - & - & - & - & - & - & - & 0.01 & - & 0.01 \\
\hline grb & - & - & - & - & - & - & - & - & - & & - & - & - & - & - & - & 0.01 \\
\hline $\mathrm{grf}$ & - & - & - & - & - & - & - & - & 0.02 & - & 0.06 & - & - & 0.02 & 0.02 & 0.07 & 0.07 \\
\hline grv & - & - & - & - & - & - & - & - & 0.06 & - & - & 0.06 & - & 0.01 & 0.35 & 0.20 & 0.20 \\
\hline gyc & - & - & - & - & - & - & - & - & - & - & - & - & - & 0.01 & - & 0.03 & - \\
\hline gyg & - & - & - & _- & - & - & - & - & - & - & - & - & - & - & - & 0.01 & - \\
\hline $\begin{array}{l}\text { hal } \\
\text { hal }\end{array}$ & 2.47 & - & 0.08 & 3.37 & 4.18 & 3.54 & - & - & 0.06 & 0.02 & 0.02 & - & 1.51 & 0.21 & 0.10 & 0.36 & 0.55 \\
\hline hay & - & - & - & - & - & - & - & - & - & - & - & - & - & - & - & - & 0.01 \\
\hline hep & - & - & - & - & _- & - & - & - & - & - & - & - & - & - & - & 0.13 & - \\
\hline hyh & - & - & 0.04 & 0.56 & 0.81 & 0.35 & - & - & - & - & - & - & 1.63 & 0.37 & 0.03 & 1.09 & 1.21 \\
\hline kar & - & - & - & - & 0.29 & - & - & - & - & - & - & 0.05 & - & - & - & 0.21 & 0.02 \\
\hline mig & - & - & 0.04 & 0.11 & - & 0.15 & - & - & - & - & - & 0.06 & 0.12 & 0.02 & - & - & 0.22 \\
\hline nip & - & - & - & - & 0.14 & 0.66 & - & - & - & - & $=$ & 0.04 & 0.21 & 0.02 & 0.46 & 0.77 & 2.16 \\
\hline phc & - & 0.01 & - & - & - & - & - & - & - & - & - & - & - & - & - & - & - \\
\hline php & 0.04 & 0.24 & - & - & - & - & - & - & - & 0.04 & - & - & - & - & 0.01 & - & - \\
\hline plc & - & - & - & 0.04 & 0.05 & 0.12 & - & - & - & - & - & - & 0.08 & 0.01 & - & 0.02 & 0.02 \\
\hline poh & 0.02 & - & 0.08 & - & - & - & 0.26 & - & - & 0.02 & - & 0.20 & - & - & - & - & - \\
\hline poe & 0.05 & - & 2.31 & - & 0.06 & - & - & 0.11 & 0.17 & 0.09 & 0.92 & 0.78 & - & - & 0.01 & - & - \\
\hline pof & 34.67 & 34.74 & 67.30 & 2.90 & 9.13 & 4.80 & 2.01 & 1.92 & 36.80 & 53.05 & 33.86 & 40.26 & 7.63 & 0.49 & 0.01 & 0.59 & 0.98 \\
\hline porn & - & - & - & - & - & - & - & - & - & - & - & - & - & - & - & 1.18 & 1.76 \\
\hline pon & - & - & 0.05 & - & - & - & - & - & - & 0.62 & 0.42 & 0.05 & - & - & - & 0.45 & 0.20 \\
\hline pou & 32.41 & 15.00 & 13.54 & 2.00 & 0.17 & 0.18 & 0.03 & - & 0.35 & 1.29 & 2.15 & 0.32 & 7.63 & 0.12 & 0.28 & - & - \\
\hline plá & - & - & - & - & - & - & - & - & - & - & - & - & - & - & - & 0.01 & 0.01 \\
\hline pte & - & - & - & - & - & - & - & _- & - & -. & - & - & - & 0.01 & - & - & 0.01 \\
\hline rhd & - & - & - & 0.05 & 0.91 & 0.62 & - & - & - & - & - & - & 0.63 & 0.07 & 0.09 & 0.09 & 0.45 \\
\hline ryt & 0.25 & 0.09 & - & - & - & - & - & - & - & - & - & - & - & - & - & - & - \\
\hline soc & 0.31 & 0.28 & 0.31 & - & - & 0.15 & - & - & 0.08 & 0.07 & 0.51 & 0.07 & - & - & - & 0.02 & 0.02 \\
\hline $\mathrm{spc}$ & 0.04 & - & - & - & - & - & - & - & - & - & - & - & - & - & - & - & - \\
\hline spm & - & - & - & - & - & - & - & - & - & -- & - & - & - & - & - & - & - \\
\hline $\mathrm{spf}$ & 0.03 & - & - & 0.02 & - & - & - & - & - & - & - & - & - & 0.01 & 0.01 & 0.07 & 0.06 \\
\hline sti & 0.07 & - & - & 0.10 & 0.12 & 0.73 & - & - & - & - & - & - & 0.43 & 0.19 & 0.90 & 0.73 & 1.01 \\
\hline$N$ & 20 & 16 & 16 & 15 & 17 & 18 & 4 & 4 & 9 & 10 & 11 & 13 & 15 & 22 & 21 & 25 & 30 \\
\hline $\mathrm{B}$ & 73.25 & 52.88 & 26.05 & 19.60 & 22.08 & 24.71 & 2.50 & 2.30 & 38.91 & 56.20 & 40.49 & 10.64 & 32.55 & 5.54 & 7.16 & 19.56 & 24.98 \\
\hline $\mathrm{SB}$ & & 75.72 & & & 22.13 & & & & & 17 & & & & & 22.45 & & \\
\hline
\end{tabular}


Table 2 (continued)

\begin{tabular}{|c|c|c|c|c|c|c|c|c|c|c|c|c|c|c|c|c|}
\hline \multirow{2}{*}{$\begin{array}{l}\text { Species } \\
\text { code }\end{array}$} & \multicolumn{4}{|c|}{$\operatorname{Stn} 7$} & \multicolumn{5}{|c|}{$\operatorname{Sin} 8$} & \multicolumn{4}{|c|}{$\operatorname{Stn} 9$} & \multicolumn{3}{|c|}{$\operatorname{Stn} 10$} \\
\hline & 71 & 72 & 73 & 74 & 81 & 82 & 83 & 84 & 85 & 91 & 92 & 93 & 94 & 101 & 102 & 103 \\
\hline $\mathrm{clp}$ & - & - & - & - & 0.03 & - & - & - & - & - & - & - & - & - & - & - \\
\hline cla & - & - & - & - & - & - & - & - & - & - & - & - & - & 0.02 & 0.01 & 0.02 \\
\hline ent & - & - & - & - & - & 0.03 & 0.01 & - & - & 0.02 & - & - & - & 0.13 & 0.17 & 1.05 \\
\hline ulv & 0.20 & 0.23 & 0.06 & 0.16 & 0.11 & 0.06 & 0.02 & 0.80 & 0.04 & 2.14 & 0.75 & 4.01 & 2.03 & 4.91 & 9.16 & 6.46 \\
\hline asp & - & - & - & - & - & - & - & - & - & - & - & - & - & - & - & 0.04 \\
\hline cop & - & - & - & - & - & - & - & - & - & - & - & 0.02 & - & - & - & - \\
\hline cum & - & - & - & - & 0.01 & 0.01 & 0.01 & 0.09 & 0.07 & - & 0.01 & - & - & 0.20 & 0.19 & 0.17 \\
\hline $\mathrm{del}$ & - & - & - & - & - & 0.06 & 0.11 & - & - & - & - & - & - & - & - & - \\
\hline did & 0.04 & 0.09 & 0.03 & 0.17 & 0.02 & 0.90 & 4.86 & 0.12 & 0.23 & 0.85 & 0.70 & 2.20 & 1.79 & 0.07 & 0.09 & 0.01 \\
\hline ect & - & - & - & - & - & - & - & - & - & - & - & - & - & 0.36 & 0.21 & 0.02 \\
\hline las & - & - & - & - & 0.45 & - & - & - & - & - & - & - & 0.10 & 1.16 & 0.60 & 0.90 \\
\hline pup & - & - & - & - & - & - & - & - & - & - & - & - & - & 0.08 & 0.10 & 0.19 \\
\hline sac & - & - & - & - & 0.32 & 0.01 & 0.03 & - & 0.03 & - & - & - & - & - & - & - \\
\hline phi & - & - & - & - & - & - & - & - & 0.03 & - & - & - & - & - & 0.04 & - \\
\hline anp & - & - & - & - & 0.18 & 0.03 & - & 0.01 & 0.01 & - & - & - & - & 0.08 & 0.01 & - \\
\hline арг & - & - & - & - & 0.01 & - & - & - & - & - & - & - & - & - & - & - \\
\hline brb & - & - & - & 0.05 & 0.68 & 1.41 & 2.29 & 8.02 & 2.82 & 1.54 & 0.79 & 1.13 & 1.78 & - & 0.25 & - \\
\hline cac & - & - & - & - & 4.50 & 0.19 & 0.42 & 0.36 & 0.09 & - & - & - & - & - & - & - \\
\hline caj & - & 0.02 & 0.05 & - & - & - & - & - & - & - & - & - & 0.02 & - & - & - \\
\hline caf & 0.06 & - & - & - & 0.13 & - & 0.10 & 0.09 & 0.01 & 0.27 & 0.01 & 0.22 & 0.02 & 0.65 & 0.21 & - \\
\hline cee & 0.03 & 0.01 & 0.07 & 0.03 & - & - & - & - & - & 1.73 & 0.76 & 3.09 & 2.62 & - & - & 0.02 \\
\hline cer & - & - & - & - & - & 0.47 & - & - & 0.03 & 2.80 & 0.80 & 1.19 & 1.52 & 6.17 & 9.87 & 7.27 \\
\hline $\operatorname{chp}$ & 0.07 & - & - & - & - & - & - & - & 0.12 & 0.02 & 0.14 & - & - & - & - & - \\
\hline chd & 0.18 & 0.60 & 0.24 & 0.62 & 0.02 & 2.23 & 3.11 & 2.73 & 1.85 & 3.46 & 2.02 & 2.84 & 3.49 & 1.10 & 1.03 & 0.70 \\
\hline chv & 0.03 & - & - & - & - & 0.19 & - & 0.18 & - & 1.54 & 1.54 & 1.61 & 1.81 & 0.02 & - & 0.09 \\
\hline coe & - & 0.02 & - & - & - & 0.13 & - & - & - & - & - & - & - & - & - & - \\
\hline $\mathrm{CII}$ & 1.07 & 0.98 & 0.66 & 1.54 & 0.40 & 0.53 & 0.30 & 0.06 & 0.10 & 1.10 & 0.50 & 1.26 & 0.76 & - & 0.09 & 0.17 \\
\hline duv & - & - & - & - & - & - & - & - & - & - & - & - & - & - & - & - \\
\hline grb & - & - & - & - & - & - & - & 0.05 & 0.02 & 0.06 & - & 0.06 & - & - & - & - \\
\hline grf & 0.04 & 0.17 & 0.07 & 0.01 & 0.02 & 0.06 & 0.50 & 0.05 & 0.03 & 0.38 & 0.90 & 1.21 & 1.35 & 0.22 & 0.32 & 0.20 \\
\hline grv & - & - & - & 0.01 & 0.03 & 0.25 & 0.82 & 0.38 & 0.83 & 3.00 & 1.44 & 2.55 & 3.52 & 2.36 & 3.48 & 2.10 \\
\hline gyc & - & - & - & - & - & 0.02 & - & - & - & - & 0.01 & - & - & - & - & - \\
\hline gyg & - & - & - & - & - & - & - & - & - & - & 0.01 & - & - & - & - & - \\
\hline hal & 0.13 & 0.03 & 0.09 & 0.06 & 0.04 & 0.13 & 0.08 & 0.25 & 0.03 & 0.53 & 0.15 & 0.25 & 0.88 & - & 0.01 & 0.07 \\
\hline hay & - & - & - & - & - & 0.01 & 0.01 & - & 0.01 & - & - & - & - & - & - & - \\
\hline hep & - & - & - & - & - & - & - & - & - & 0.02 & - & - & - & - & - & - \\
\hline hyh & - & - & 0.04 & 0.06 & - & 0.11 & - & - & - & 0.02 & 0.13 & 0.27 & 0.26 & 0.01 & 0.26 & 0.23 \\
\hline kar & - & - & - & - & 0.07 & - & - & - & - & 0.71 & 1.97 & 1.96 & 0.26 & - & - & 0.07 \\
\hline mig & 0.02 & 0.08 & 0.07 & 0.28 & - & - & - & - & - & - & - & - & - & - & - & - \\
\hline nip & 0.02 & 0.05 & 0.05 & 0.04 & - & - & 0.02 & - & - & 0.10 & 0.12 & 0.16 & 1.09 & 0.63 & - & - \\
\hline phe & - & - & - & - & - & - & - & - & - & - & - & - & - & - & - & - \\
\hline php & - & - & - & - & 0.12 & 0.03 & 0.33 & - & - & - & - & - & - & - & - & - \\
\hline plc & - & - & 0.03 & - & - & 0.87 & - & - & - & - & 0.02 & 0.02 & - & - & - & - \\
\hline poh & - & - & - & - & 45.80 & - & 0.34 & - & 0.12 & - & - & - & - & - & - & 0.04 \\
\hline poe & - & 0.13 & - & 0.10 & 0.64 & 0.06 & 0.03 & - & 0.01 & 0.04 & 0.05 & - & 0.04 & 0.21 & 0.25 & 1.41 \\
\hline pof & 2.77 & 3.13 & 1.01 & 2.86 & 17.38 & 8.39 & 8.21 & 7.27 & 3.84 & 0.74 & 1.16 & 6.72 & 7.63 & 2.78 & 1.83 & 1.92 \\
\hline pom & - & - & - & - & - & - & - & - & - & 1.20 & 0.65 & - & 1.61 & - & - & - \\
\hline pon & - & - & - & - & 0.05 & 0.14 & 0.63 & 0.43 & 0.76 & 0.65 & 0.53 & 0.53 & 1.21 & 0.02 & 0.21 & - \\
\hline pou & 0.03 & 0.05 & 0.06 & 0.25 & 0.02 & 0.41 & 0.14 & 1.19 & 0.36 & - & - & 1.85 & - & 1.42 & 0.59 & 0.67 \\
\hline pta & - & 0.04 & - & - & - & - & - & - & - & - & - & - & - & - & - & - \\
\hline pte & - & - & - & - & - & - & - & - & - & 0.02 & - & - & - & - & - & - \\
\hline rhd & - & - & - & 0.10 & - & 0.07 & 0.02 & - & - & 0.11 & 0.13 & 0.24 & 0.29 & - & 0.02 & - \\
\hline$\Gamma y t$ & - & - & - & - & - & - & - & - & - & - & - & - & - & - & - & - \\
\hline soc & - & 0.03 & - & - & 2.70 & 0.88 & 1.15 & 1.61 & 0.44 & 0.05 & 0.02 & 0.07 & 0.10 & - & - & - \\
\hline $\mathrm{spc}$ & - & - & - & - & - & - & - & - & - & - & - & - & - & - & - & - \\
\hline $\mathrm{spm}$ & - & - & - & - & 0.01 & 0.01 & 0.01 & - & - & - & - & - & - & - & - & - \\
\hline spf & - & 0.07 & 0.17 & 0.04 & 0.11 & 0.28 & 0.06 & 0.10 & 0.20 & 1.31 & 1.14 & 3.26 & 2.89 & 0.05 & 0.30 & 0.02 \\
\hline su & 0.09 & 0.11 & 0.21 & 0.25 & - & 0.21 & 0.34 & 0.12 & 0.25 & 0.35 & 0.70 & 1.23 & 0.98 & - & - & - \\
\hline $\mathrm{N}$ & 15 & 18 & 16 & 18 & 26 & 31 & 27 & 20 & 26 & 28 & 28 & 25 & 25 & 22 & 25 & 24 \\
\hline B & 4.78 & 5.84 & 2.91 & 6.62 & 73.85 & 18.18 & 23.95 & 23.97 & 12.33 & 24.75 & 17.15 & 37.95 & 38.05 & 22.65 & 29.30 & 23.75 \\
\hline $\mathrm{SB}$ & & 5. & 94 & & & & 30.45 & & & & & 48 & & & 25.23 & \\
\hline
\end{tabular}




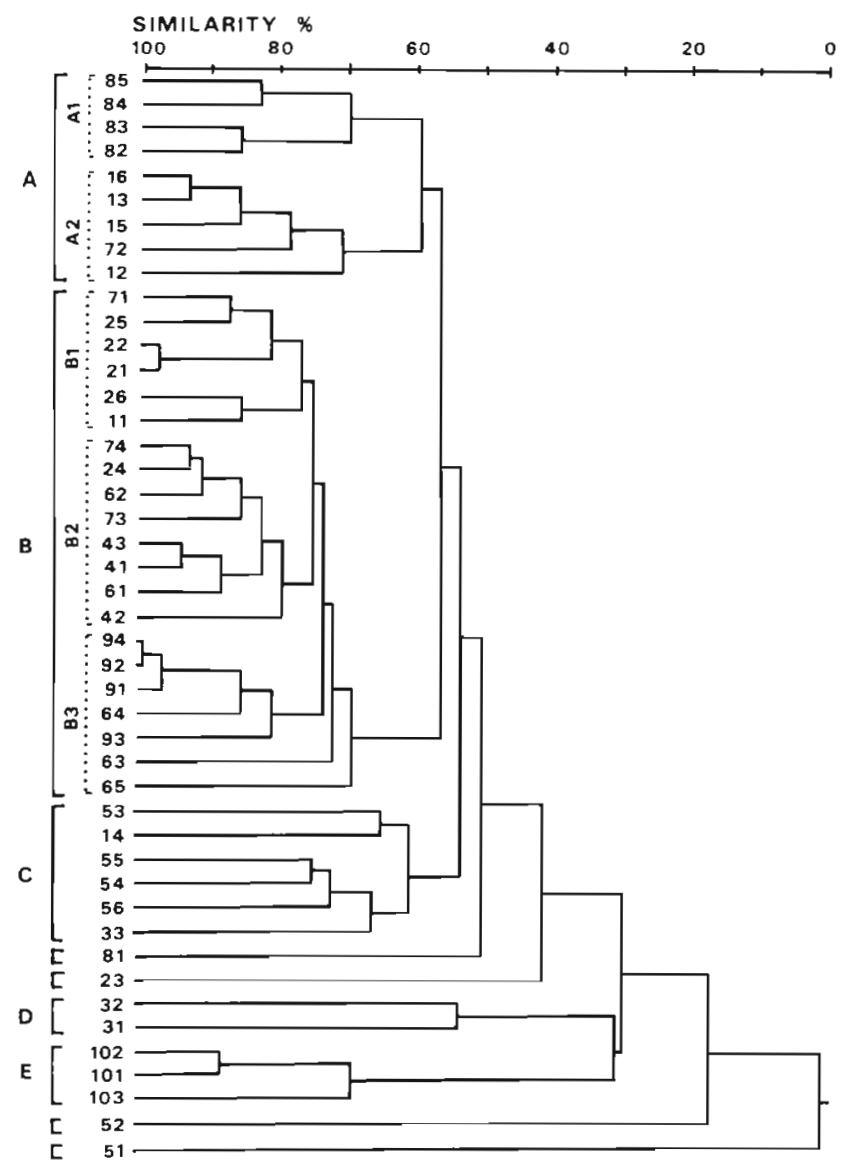

Fig. 4. Dendrogram showing classification of 45 samples based on macrophyte biomass. Dendrogram was formed by group-average sorting ( $\chi^{2}$ distance). Five main clusters (A to E) are distinguished at an arbitrary similarity level of $55 \%$ (x-axis)

(E) Ulva sp. assemblage, sandy mud without shell and living maerl.

The inverse analysis was conducted in an attempt to determine the species affinities. The dendrogram shows 2 different clusters separating at approximately $30 \%$ similarity. The main cluster subdivided into 2 others clusters at approximatively $60 \%$ similarity (Fig. 5). Some species were separated from these clusters because they were found only in a single station. Three groups of species were designated from the analysis of seaweed substrata: $(E)$ epilithic species, $(U)$ indifferent species, (M) maerl preferent species.

\section{Ordination}

Ordination by correspondence factor analysis (CFA) of samples confirmed that the assemblages are spatially structured. Results of the 2 complementary methods unanimously agreed, proving that the discontinuities enhanced by classification can be accepted as real. Clusters of samples from the classification have been delimited in Planes 1 and 2 in Fig. 6.

Samples were clearly grouped in different sectors similar to the results of the clusters analysis. The groups were situated along Axis 1 in the order $D, C, A$, and $B$ was separated from the others by Axis 2. Axis 1 represents graphically a gradient of decreasing hydrodynamic activity (from the left side to the right side) represented by modifications of the nature of substrata. Axis 2 represents graphically the role played by the nature of substrata on the algal assemblage composition and particularly the maerl quality for the group B.

The contribution to the total inertia of the first and second axes were 25.2 and $13.3 \%$ respectively. Major absolute contributors to the inertia explained by Axis 1 were, in decreasing order, Solieria chordalis C. Agardh, Polysiphonia elongata Hudson, Polysiphonia urceolata Lightfoot. ex Dillwyn., Polysiphonia fibrillosa

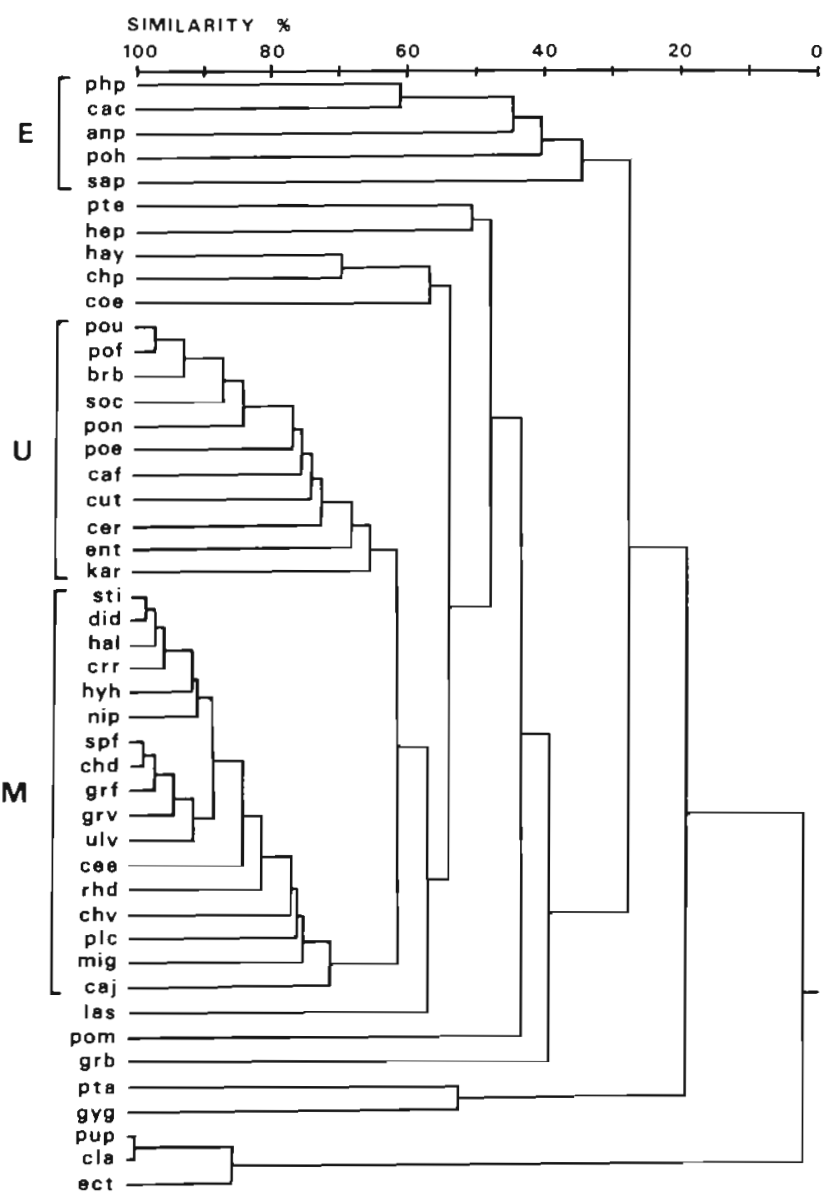

Fig. 5. Dendrogram of inverse analysis comparing 46 macrophytes species occuring in the 45 samples of the survey. Dendrogram was formed by group-average sorting $\left(x^{2}\right.$ distance). Three main groups are distinguished at an arbitrary similarity level of $50 \%$. Rare species are thrown out of these groups 


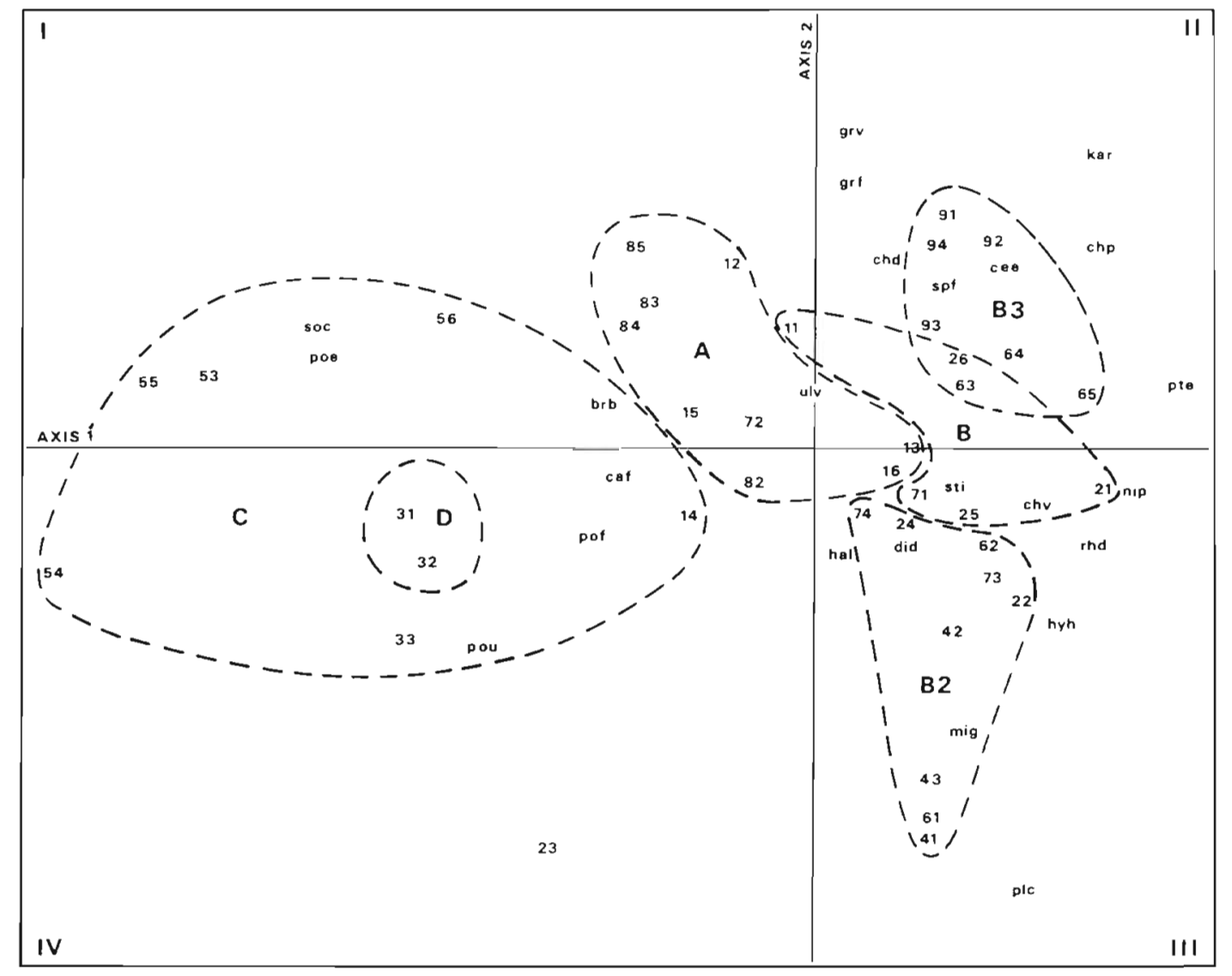

Fig, 6. Correspondence factorial analysis graph. Projection of variables (macrophyte species) and observations (samples) in Planes 1 and 2. After a first analysis rare species and very isolated samples $(51,52,81,101,102,103)$ were removed. Contribution to the total inertia of the first axis was $25.22 \%$, and second axis $13.33 \%$. Major absolute contributors to the inertia explained by Axis 1 were, in decreasing order, variables: Solieria chordalis, Polysiphonia elongata, Polysiphonia urceolata; observations: 54, 55, 53. For Axis 2 major absolute contributors were variables: Gracilaria verrucosa, Plocamium cartilagineum, Gracilaria folifera; observations: 61, 41,91. Each species is represented by the first 2 letters of the genus name and the first letter of the species name (e.g. Polysiphonia fibrillosa $=$ pof, see also Table 2)

Dillwyn, Nitophyllum punctatum Stackhouse, and Brongniatella byssoides Goodeviough et Woodward. For Axis 2, major absolute contributors were Gracilaria verrucosa Hudson, Plocamium cartilagineum Linnaeus, Gracilaria foliifera Forskal, Polysiphonia urceolata and Microcladia glandulosa Solander ex Turner. Projection of the observations (samples) on the CFA plane were correlated with those of variables represented here by species. Distribution of the latter shows a clear relation with the substratum and the hydrodynamic activity as previously shown by the delineation of dendrograms clusters.

\section{Faunal assemblages}

\section{Richness and diversity}

The highest total number of species, $S$, was observed on the Banc du Corbeau (Stns 3 and $8: S \geq 40$ ) in the western site (HES), and the lowest in the DEL and SHM (Stns 7, 9 and 10: $S<25$ ) (Table 3). The Lithothamnion beds DLL and MIL were homogenous with intermediate values of $S=30$ ). Three species were locally dominant and induced high abundance $(A)$ or biomass $(B)$ and low $H^{\prime}$ values: Balanus crenatus Bruguière (Stn 7: $A>500 \mathrm{~m}^{-2}$ ), Crepidula fornicata (Stns 2, 3 and $7: A>50 \mathrm{~m}^{-2}$ ), Ficulina ficus (Stn 5: $B>$ $\left.400 \mathrm{~g} \mathrm{~m}^{-2} ; H^{\prime}=0.7\right)$. A total of 110 different species were recorded at the 10 stations.

\section{Trophic structure}

Faunal data were analyzed by the same methods as the algal data. Four clusters of samples were identified by the classification analysis (A, B, C, D: similarity $=60 \%$ ). Four secondary clusters were individualized in the $A$ group $\left(A_{1}\right.$ to $A_{4}: 70 \%$ homologous similarity). The grouping of samples and stations 
Table 3. Richness of the fauna in the study area in terms of no. of individuals, no. of species, biomass and specific diversity $\left(H^{\prime}\right.$, Shannon index). Average values (SE) of 5 samples

\begin{tabular}{|c|c|c|c|c|c|c|c|c|c|}
\hline \multirow{2}{*}{$\frac{\text { Stn }}{1}$} & \multicolumn{2}{|c|}{$\begin{array}{l}\text { Abundance } \\
\text { (ind. } \mathrm{m}^{-2} \text { ) }\end{array}$} & \multicolumn{2}{|c|}{$\begin{array}{c}\text { No. of } \\
\text { species } \mathrm{m}^{-2}\end{array}$} & \multicolumn{2}{|c|}{$\begin{array}{l}\text { Biomass } \\
\left(\mathrm{g} \mathrm{m}^{-2}\right)\end{array}$} & \multicolumn{2}{|c|}{$\begin{array}{c}\text { Diversity index } \\
\left(H^{\prime}\right)\end{array}$} & \multirow{2}{*}{$\begin{array}{c}\text { Total no. o } \\
\text { species, } S \\
36\end{array}$} \\
\hline & & (14) & 14.1 & $(3.0)$ & 10.4 & $(5.8)$ & 1.40 & $(0.65)$ & \\
\hline 2 & 99 & (83) & 14.2 & $(3.4)$ & 49.3 & $(29.3)$ & 2.5 & $(0.9)$ & 29 \\
\hline 3 & 101 & $(102)$ & 17.2 & $(2.2)$ & 20.8 & $(14.2)$ & 1.80 & $(0.56)$ & 46 \\
\hline 4 & 70 & (34) & 15.3 & $(3.1)$ & 9.7 & $(6.0)$ & 2.80 & $(0.27)$ & 28 \\
\hline 5 & 14 & (7) & 2.0 & $(1.8)$ & 429 & $(137)$ & 0.70 & $(0.26)$ & 7 \\
\hline 6 & 69 & (39) & 12.6 & $(4.8)$ & 224 & (221) & 3.17 & $(0.40)$ & 27 \\
\hline 7 & 568 & $(175)$ & 11.0 & $(3.6)$ & 13.6 & (9.5) & 2.50 & $(0.35)$ & 23 \\
\hline 8 & 279 & $(480)$ & 14.4 & $(2.6)$ & $<5$ & - & 2.7 & $(0.5)$ & 40 \\
\hline 9 & 83 & (21) & 11.7 & $(3.2)$ & $<5$ & - & 3.90 & $(0.06)$ & 25 \\
\hline 10 & 11 & (11) & 7.3 & $(0.9)$ & $<5$ & - & 2.90 & $(0.15)$ & 11 \\
\hline
\end{tabular}

obtained by the fauna analysis did not differ much from that obtained by algal analysis (cf. Fig. 4). Analysis of the trophic structure according to these clusters (Fig. 7) shows a progressive substitution of suspension feeders by herbivores. Thirty-eight suspension-feeding species were encountered including the sponges Halichondria panicea Pallas and Ficulina ficus Olivi, the polychaetes Potamoceros triqueter Linnaeus and Serpula vermicularis Linnaeus, the gastropod Crepidula fornicata Linnaeus, many bivalves such as Anomia ephippium Linnaeus and Modiolus barbatus Linnaeus, the crustacean Balanus crenatus Bruguière, and numerous ascidians (Ascidia mentula Muller, Phallusia mammillata Cuvier, Pyura microcosmus Savigny, Morchellium argus Milne-Edwards). Herbivores consisted of gastropods (Aplysia punctata Cuvier, Calliostoma zizifinum Linnaeus, Cantharidus striatus Linnaeus, Gibbula magus Linnaeus, Gibbula cineraria Linnaeus), polyplacophores (Achantochitona crinatus Pennant, Tonicella marmorata Fabricius), and echinoderms (Echinus esculentus Linnaeus, Psammechinus miliaris Gmelin, Spherechinus granularis Lamark). Carnivores (essentially small crustaceous Decapods and Gastropods) and deposit feeders (Plathynereis dumerilii Audouin and Milne-Edwards, Eunice harassi Audouin and Milne-Edwards) had low variations in the different clusters (dominance varied from 20 to $30 \%$ ).

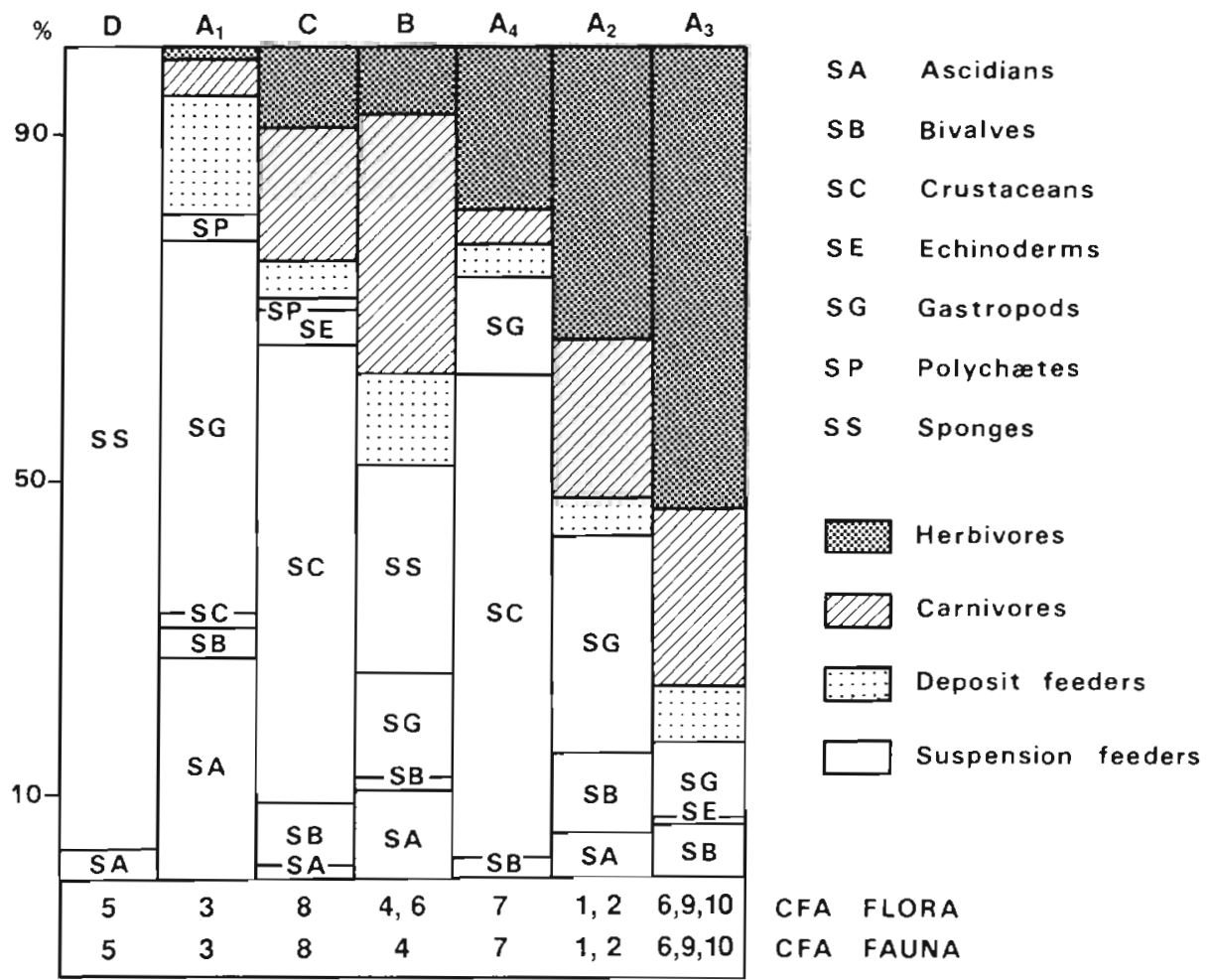

Fig. 7. Trophic structure of epifauna in the groups of samples identified by ordination analysis on total faunal data. Main sampling stations of each group are indicated on the last 2 lines of the figure. Clusters of samples identified by algal and by faunal analyses were found to be very similar. CFA = Correspondence Factorial Analysis 
Role of fauna in the quantitative and qualitative variability of supports

Analysis of algal support characteristics in each cluster (Table 4) illustrated the major role of fauna in supplying algae with substrata. After the death of the animals, calcareous structures (shells, tubes) settle on the sediment surface and become suitable substrata for algal fixation and growth. These biogenic supports constituted more than $80 \%$ of the total number of supports in Groups B1, B2, and E, and more than $50 \%$ in Groups B3 and D. Moreover, they differed qualitatively from one group to another: $48.75 \%$ of the supports were Crepidula shells in Group A whereas $44.22 \%$ were bivalve shells in Group B3 and $40.55 \%$ small gastropods (Gibbula, Calliostoma) in Group E. This variability of biogenic substrata is a reflection of the living population's diversity.

\section{DISCUSSION}

\section{Dissimilarities in algal assemblages}

Algal assemblages were largely different from the assemblages described by Cabioch (1969) for the maerl beds of the Bay of Morlaix (north Brittany, France). The main assemblage dominated by Solieria chordalis, Halopytis incurvus, Rytiphlea tinctoria (Clemente) was not found in the Bay of Brest. The turbidity which has globally increased over the last $10 \mathrm{yr}$ (Hily 1984) can explain this main alteration of the algal communities in the northern basin of the Bay of Brest. Only $S$. chordalis was recently found to be abundant in calm waters of the southern basin (Floc'h et al. 1987). Modifications of agricultural activities which enhanced the terrigenous effluents by the rivers are responsible for this increase in turbidity. The assemblage observed on maerl beds in the summer was not far from the other maerl asemblages in Brittany, but the Pheophyceae remained rare [Rhodophyceae : Pheophyceae $=1: 10$ in the Bay of Brest, while this ratio was $1: 2$ in Glenan Archipelago, southern Brittany (Blunden et al. 1977)].

\section{Algal production}

The annual production of macrophytes in the study area cannot be easily calculated from these experiments because the biomass variations over time were not surveyed. However, a rough estimation can be made considering only the 4 mo period from $t_{1}=$ March, biomass $B_{1}=0$, to $t_{2}=$ June, biomass $B_{2}=$ new production. From biomass values (Table 2, biomass station $^{-1}$ ) and their extrapolation to the whole area, which was divided in 5 assemblages (according to the cluster results and the sedimentary units; Fig. 2), the mean production should be $6 \mathrm{~g} \mathrm{C} \mathrm{m}^{-2}$. Carbon : dry organic matter retained was $30 \%$ (value obtained from 18 macrophytes of Brittany by Kerambrun 1984).

\section{Factors controlling algal assemblages}

Analysis of algal distribution on the soft bottoms of the study area showed a succession of assemblages on a west-east gradient. Spatial distribution of algal assemblages is the result of the positive and negative factors influencing the biological parameters of the different species. The abiotic factors appeared as the first group of factors controlling the structure and the specific composition of assemblages and particularly hydrodynamic activity, which acts as a disturbance. But the biotic factors such as interspecific competition, fau-

Table 4. Qualitative and quantitative characteristics of the supports used by algae in the groups of stations defined by algal analysis. Standard error given in parentheses

\begin{tabular}{|c|c|c|c|c|c|c|c|c|}
\hline \multirow[b]{3}{*}{ Characteristic clusters: } & \multicolumn{8}{|c|}{ Sampling station } \\
\hline & \multicolumn{2}{|c|}{$8-1$} & 2 & $4-6$ & 9 & 5 & 3 & 10 \\
\hline & \multicolumn{2}{|c|}{$\mathrm{A} 1-\mathrm{A} 2$} & B1 & B2 & B3 & $\mathrm{C}$ & $\mathrm{D}$ & E \\
\hline Total no. of supports & \multicolumn{2}{|c|}{$39(40)$} & $44(24)$ & $4(1)$ & $27(13)$ & 55 & $82(2)$ & $19(2)$ \\
\hline Mineral support (\%) & \multicolumn{2}{|c|}{54.18} & 8.38 & 20 & 34.8 & 72.5 & 49.82 & 10.18 \\
\hline Crepidula shells (\%) & \multicolumn{2}{|c|}{3.4} & 48.75 & 40 & 12 & 0 & 9.37 & 17.65 \\
\hline Other Gastropoda shells (\%) & \multicolumn{2}{|c|}{2.3} & 2.66 & 0 & 9.59 & 2.25 & 16.2 & 40.55 \\
\hline Bivalvia shells $(\%)$ & \multicolumn{2}{|c|}{32.29} & 15.84 & 6.67 & 44.22 & 4.3 & 4.69 & 31.62 \\
\hline Polychaeta tubes $(\%)$ & \multicolumn{2}{|c|}{7.82} & 17.25 & 37.33 & 0.28 & 20.7 & 20.7 & 0 \\
\hline Total animal biogenic supports $(\%)$ & \multicolumn{2}{|c|}{45.82} & 91.62 & 80 & 65.2 & 27.5 & 50.18 & 89.82 \\
\hline \multirow[t]{2}{*}{ Surface covered by living Lithothamnium (\%) } & A1 & A2 & & & & & & \\
\hline & 0 & 47 & 0 & 98 & 30 & 20 & 17 & 0 \\
\hline \multirow[t]{2}{*}{ Surface covered by dead Lithothamnium (\%) } & A1 & A2 & & & & & & \\
\hline & 0 & 47 & 80 & 0 & 30 & 0 & 0 & 75 \\
\hline
\end{tabular}


nal/floral competition, grazing by herbivores, and animal contribution in substrata must be taken into account to understand the mechanisms by which the main disturbance acts. Disturbance can be considered in a variety of ways, according to the spatial and temporal scale of observation: (1) Clements (1936) and Grime (1977) defined disturbance to be a negative force that destroys climax assemblages, inducing an unstable state. (2) Paine (1966) and Lubchenco \& Menge (1978) considered the disturbance to be a positive force, preventing competitive exclusion by dominant species and consequently increased diversity. (3) Disturbance should be a necessary feature of community dynamics which maintains a regionally stable state (Sprugel 1976). We consider these different points of view to be complementary, each of them serving to explain a part of the results.

\section{Abiotic factors}

Temperature and salinity can be considered to have negligible action because they have low variations within the whole area during the production period of algae. On the other hand, hydrodynamic activity (i.e. tidal currents, waves and turbidity) showed large variations of intensity in space and was certainly the main factor controlling species distribution.

\section{Tidal currents}

Tidal currents remain slow in the greater part of the study area, with the exception of the Elorn River estuary ( $\operatorname{Stn} 9)$, where they can reach more than $1 \mathrm{knot} \mathrm{h}^{-1}$ at spring tide and increase resuspension and disturbance by waves. However, disturbance caused by tidal currents remains low compared to that of waves.

\section{Waves}

In the western part of the study area, the swell, frequent in autumn and winter, turns cobbles and carries shells and sand. Persistence of a stable algal community is impossible in this area, only crustose algae can remain throughout the year. These features reveal, according to Davis \& Wilce (1987a), communities controlled by physical factors in which tidal currents and storms have sufficient force to cause alteration in the substrata. Instability of substrata allowed only the development of opportunistic species such as polysiphonia, which grows very rapidly in the spring and reaches a high biomass $\left(1000 \mathrm{~g}\right.$ organic wet wt $\left.\mathrm{m}^{-2}\right)$. Diversity was low (Shannon index $H^{\prime}<1.5$ ); Stns 3, 5 and 9 were examples of this. Support disturbance can create free patches of space through the elimination of certain substrata and associated algae by transport (Davis \& Wilce 1987a), and enhance the variability of abundance and diversity within one sampling station. Moreover, inter- and intra-station heterogeneity existed because, according to Sousa (1979), it is the frequency with which a support is disturbed, rather than simply its size, that is responsible for different patterns in the species composition in substrata of different stability. Consequently due to the heterogeneity of disturbance frequency, and the size and nature of the substrata, the species composition is unpredictable in small temporal and spatial scales. In a larger scale, estimation of a mean frequency of the support disturbance can determine the time interval over which the successive species replacement can occur. However, as highlighted by Sousa (1979), the successive age of a boulder does not return to zero each time it is overturned by waves; often some organisms survive and continue to grow, a fact which increases heterogeneity between boulders. This explains the observed complexity of the species composition and production. When hydrodynamic activities are decreasing (Stns 2, 4 and 6) maerl is able to grow. Maerl beds were stabilized by a persistant epiflora (Gelidiella calcicola Maggs et Guiry, Dictyocta dichotoma Hudson), which produces adventitious links between the maerl shrubs. Several selective species characterize the 'maerl assemblage'.

\section{Turbidity}

A transitional area with living and dead maerl exists in the eastern study area (Stns 1 and 7). Growth of maerl is limited by the turbidity induced by the terrigenous effluents of the Elorn River, the second main abiotic factor controlling algal assemblages. Dead maerl is poorly colonized by epiflora and can be easily carried by currents and waves to the eastern part of the bay. Biomass remains low (Table 2, Stn 7). Species found were those which constitute the maerl beds and the ubiquitous ones (Rhodophyceae and Pheophyceae).

Stn 9 is situated on a bank which was previously exploited for oyster beds (Ostrea edulis) before the high mortalities which affected this species in the $1960 \mathrm{~s}$. Large amounts of shells remain on the bottom. The contribution of the bivalves as substrata for epiflora and epifauna is now enhanced by 2 bivalve populations: the black scallop Chlamys varia Linnaeus and Venerupis pullastra Montagu. The algal assemblage is composed of the ubiquitous species (Rhodophyceae and Pheophyceae), which are not controlled by the high turbidity (usually exceeding $800 \mathrm{mg} \mathrm{l}^{-1}$ ). 
Turbidity determined the vertical distribution of macrophytes in the eastern part of the study area (Stn 10), which was dominated by Ulva sp; at depths greater than $2 \mathrm{~m}$, biomass decreased very rapidly from 10 to $1 \mathrm{~g} \mathrm{~m}^{-2}$. In this sheltered bay, eutrophication favored Chlorophyceae development and particularly the Ulva, which can grow free of substrate. The lack of residual current in the bay is another favorable factor for the development of Ulva (Menesguen \& Monbet unpubl.). Competition with other species is minimal because of the very low densities of favorable supports for fixed algae. The bottom was essentially covered by small fragments of dead maerl in which the Rhodophyceae fixation is rare and temporary.

\section{Biotic factors: faunal/floral interactions}

Biotic factors influenced the algal assemblages in 2 ways: (1) directly, by spatial competition with fauna and grazing by herbivores and (2) indirectly, by contributing animals to the shelly substrata.

\section{Direct relationships}

Spatial competition between fauna and flora was not a major factor of control. At Stn 5, however, this phenomenon was important because of the abundance of large suspension feeders (Ficulina ficus and Phallusia mammillata) which commonly occupied more than $50 \%$ of the sediment surface. Opportunistic species such as Polysiphonia fibrillosa and $P$. urceolata have the best adaptability in this competition. This particularity explains the isolated situation of Stn 5 in the dendrograms. A similar competition between algae recruitment and suspension feeders has been observed by Dayton (1975) in the intertidal zone in California (USA) between the Mytilus edulis Linnaeus mats and the Lessonopsis algae. Dethier (1984) studied the disturbance and recovery in intertidal pools and enhanced the role of Mytilus californiensis and Anthopleura elegantissima as spatial competitors of algae. At the normal herbivore densities, as demonstrated by Dayton (1975), the growth of algae restricts the ability of the herbivores to eat the young plants, therefore the plants are no longer threatened. The predation by decapods and Asterias rubens Linnaeus maintained the densities of most molluscan and echinoderm herbivores below the presumed capacity of the environment. In the Bay of Brest, the presence of Sphaerechinus granularis Lamark, reaching local densities of 2 to 3 ind. $\mathrm{m}^{-2}$, can affect the algal cover in small spatial and temporal scales. But this should not affect the control of the algal cover in the scale of the whole area by limiting biomass and by modifying the specific composition assemblage as a biotic disturbance. On the other hand, hydrodynamic activity was a disturbance for the herbivores via algae in 2 ways: (1) many small molluscan herbivores living on the macrophytes are carried with their supports to the eastern part of the bay and driven ashore in autumn and winter. Despite the high abundance and biomass of the Aplysia punctata population, the proliferation of Ulva is not biologically controlled by grazing because each storm carries to shore a high biomass of Ulva and Aplysia. (2) The lack of algal feeding throughout the year is a limiting factor which prevents the establishment of a structured and persistent assemblage of animal species dominated by the herbivorous species (Fig. 7, Stns 5, 3, 8, 4 and 6). The decrease of hydrodynamic disturbance to the east of the bay is correlated to a progressive increase in dominance of herbivores in the epifaunal trophic structure. In fact, the abundance of the macro-detritus of algae throughout the year in the eastern areas allows the persistence of herbivores outside the period of new macrophyte production.

\section{Indirect relationships}

Indirect relationships between algae and fauna are linked with the algal dependance on the supports for recruitment and growth. Except at Stns 3 and 8 (Table 4), almost all algal supports were biotic supports, i.e. shells of bivalves and gastropods, calcareous tubes of polychaetes. Most of these supports are transported by waves and currents when growth of the fixed algae increases the buoyancy of the 'algae + shell' system. The population dynamics parameters (particularly the mortality) of the shell species determine the densities and the qualitative characteristics of the substrata. Size and quality of the animal's biotic supports are also important in the qualitative composition of the algae assemblages. Liebermann et al. (1984) acknowledged this fact from the results of Harlin \& Lindberg (1977) which demonstrated that the difference in availability of spores is influenced by surface texture and microtopography. The small substrata remain in the early stages of colonization with an abundance of open space and are usually dominated by those species settling in the highest abundances in that one period of time (Sousa 1979). In the Bay of Brest these opportunistic species were the cirripedian Balanus crenatus which covered most of the small supports and competed for space with the opportunistic algae Polysiphonia fibrillosa and P. urceolata.

Dredging can also be considered as an anthropogenic disturbance of algae, supports and epifauna. The black scallop Chlamys varia is locally abundant on 
these banks and is intensely fished by dredging in the winter period. As demonstrated before (Hily \& Le Foll 1990), this process induces large modifications in the substrata distributions.

\section{Disturbance and limiting factor}

The spatial patterns of disturbances and limiting factors in algal assemblages are shown in Fig. 8. A

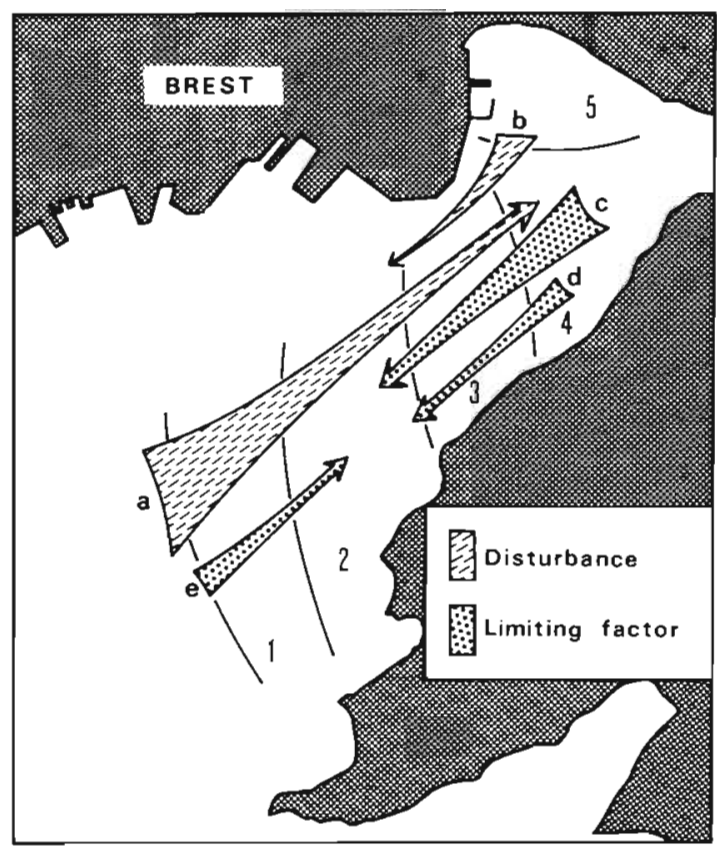

Fig. 8. Main disturbances and limiting factors of algal assemblage distribution in the northern part of the Bay of Brest. (a): wave disturbance; (b): grazing; (c): turbidity; (d): shelly substrate availability; (e): spatial competition; (1) to (5): algal assemblages (described in text)

succession of 5 units identified by algal and faunal assemblage analysis is situated in a SW to NE gradient. Hydrodynamic activity, particularly waves (a), and grazing by herbivores (b), which destroy biomass, can be considered as disturbances according to the definition of Grime (1977) (see 'Introduction' of this paper); they are in opposition in this gradient. Turbidity (c) and density of animal substrata (d), which prevent development of some species and restrain production, can be considered as limiting factors; in the gradient they are in synergy and opposed to the 'spatial competition' factor (e) (i.e. spatial competition between suspension feeders and algae).

Sutherland (1981) described 3 types of disturbances: Type I - those that are not capable of changing the resident assemblage (the community has persistent stabil- ity); Type II - those that alter community structure, but only temporarily (so that it readjusts within several generations); Type III - those that change the resident assemblage 'permanently'. In Type III the global stability is unattainable, and is a relative term. The situation observed in algal assemblages of the Bay of Brest can be interpreted as a succession of the 3 described types of disturbances: the resident assemblage of the western area is always dominated by opportunistic species and the global stability is unattainable due to the high frequency of hydrodynamic disturbances (Type III) (Fig. 8, Unit 1). In the middle area the maerl bed assemblage is altered only in autumn and winter, during the highest period of storms and gales. The biomass is temporarily destroyed but the species assemblage is readjusted the following spring (Type II) (Fig. 8 , Units 2 and 3 ). In the eastern area the assemblage is dominated by long-living species which are controlled by limiting factors, such as turbidity, rather than hydrodynamic and grazing disturbances, which cannot deeply alter the assemblage structure (Type I). Dredging for the black scallop is a Type II disturbance, superimposed locally in the 3 areas (Fig. 8, Units 4 and 5).

\section{CONCLUSION}

This study showed that methods of ecological analysis classically utilized in animal ecology can be used efficiently to analyze the algal ecology. The similar steps taken for the analysis of floral and faunal data in the same area and period of time is not frequent in literature, but provides interesting conclusions about the ecology of shallow, soft-bottom sediments. It demonstrates the necessity of integrating the physical/ floral/faunal processes in order to understand the functioning of the shallow-bottom ecosystems. The distribution of algae is the result of abiotic and biotic factors.

Abiotic factors acted as stochastic (waves) and periodic (tidal currents) disturbances (Sousa 1979) which destroy biomass. On the other hand, the same factors operated as a positive force which increased the production and the productivity of the algal fields. In the same way, disturbance is important in providing space for colonization by opportunistic species and in maintaining high diversity assemblages.

The fauna/flora interactions also provided positive and negative impacts upon the populations. Grazing by herbivores reduced algal biomass but enhanced its production. Persistence of algal cover increased the biomass and diversity of herbivores and associated carnivores. Suspension feeders and algae were in competition for occupation of space, but algae provided detritus exploited by the suspension feeders during the winter periods. 
According to the 'niche concept' of Hutchinson (1957) which is defined as 'the set of conditions in which the species live and breed', we can consider that the ecological niche of Lithothamnium corallioides, which determines the maerl assemblage of erect macrophytes, is very narrow and subjected to many controlling environmental factors. Moderate current and wave action on the one hand, but moderate turbidity and sedimentation on the other, are antagonistic conditions which explain the limited ecological area and spatial distribution observed for this species on the soft, shallow-bottom of the coastal temperate waters.

Few studies have examined the relationships between algal diversity and the stability of substrata in marine environments (Lieberman et al. 1979, 1984, Sousa 1979a). This paper demonstrated that the development of macrophyte assemblages on soft-bottom sediments was almost entirely dependant on substrata which was only biotic (either calcareous algae as maerl or shells of benthic invertebrates). Variability of algae populations was then correlated with the dynamics of the populations which played the role of substrata (maerl, bivalves, gastropods). Finally the availability of those substrata was controlled by the physical disturbance of waves and tidal currents.

Acknowledgements. The authors thank Mrs Margaret Pratt who corrected the English version of the manuscript. The study was realized in the laboratoire d'Océanographie Biologique and the Laboratoire de Physiologie Végétale of the Université de Bretagne Occidentale, Brest, France.

\section{LITERATURE CITED}

Benzecri, J. P. (1973). L'analyse des données. Tome 1: la taxinomie. Tome 2: l'analyse des correspondances. Dunod Ed., Paris

Blunden, G., Farnham, W. H., Jephson, R. H., Fenn, R. H., Plunkett, B. A. (1977). The composition of maerl from the Glenan Islands of southern Brittany. Bot. Mar. 20: 121-125

Blunden, G., Farnham, W. H., Jephson, R. H., Barwell, C. J., Fenn, R. H., Plunkett, B. A. (1981). The composition of maerl beds of economic interest in northern Brittany, Cornwall and Ireland. Proc. int. Seaweed Symp. 10: $651-656$

Cabioch, J. (1966). Contribution à l'étude morphologique, anatomique et systématique de deux Mélobésiées: Lithothamnium calcareum (Pallas) Areschoug et Lithothamnium corralioides Crouan. Bot. Mar. 9: 33-53

Cabioch, J. (1969). Les fonds de maerl de la baie de Morlaix et leur peuplement végétal. Cah. Biol. Mar. 10: 139-161

Clements, F. E. (1936). Nature and structure of the climax. J. Ecol. 24: 252-284

Davis, A. N., Wilce, R. T. (1987a). Algal diversity in relation to physical disturbance: a mosaic of successional stages in a subtidal cobble habitat. Mar. Ecol. Prog. Ser. 37: 229-237

Davis, A. N., Wilce, R. T. (1987b). Floristics, phenology, and ecology of the sublittoral marine algae in an unstable cobble habitat (Plum Cove, Cape Ann, Massachusetts, USA). Phycologia 26(1): 23-34
Dayton, P. K. (1975). Experimental evaluation of ecological dominance in a rocky intertidal algal community. Ecological Monogr. 45: 137-159

Dethier, M.N. (1984). Disturbance and recovery in intertidal pools: maintenance of mosaic patterns. Ecol. Monogr. 54 (I): $99-118$

Fichaut, B. (1984). Réactualisation de la sédimentologie de la rade de Brest. Thèse de $3^{\text {ème }}$ cycle, Univ. Bretagne Occidentale, Brest

Field, J. G., Clarke, K. R., Warwick, R. M. (1982). A practical strategy for analysing multispecies distribution patterns. Mar. Ecol. Prog. Ser. 8: 37-52

Floc'h, J Y., Deslandes, E., Le Gall, Y. (1987). Evidence for a vegetative propagation of the carraghenophyte Solieria chordalis (Solieraceae, Rhodophyceae) on the coast of Brittany (France) and in culture. Bot. Mar. 30: 315-321

Frontier, S. (1976). Utilisation des diagrammes rangsfréquences dans l'analyse des écosystèmes. J. Rech. Océanogr. 1(3): 35-48

Grime, J. P. (1977). Evidence for the existence of three primary strategies in plants and its relevance to ecological and evolutionnary theory. Am. Nat. 111: 1169-1194

Harlin, M. M., Lindberg, J. M. (1977). Selection of substrata by seaweeds: optimal surface relief. Mar. Biol. 40: $33-40$

Hily, C. (1983). Modifications de la structure écologique d'un peuplement de Melinna palmata (Annélide Polychète) soumis aux effluents urbains et industriels en rade de Brest. Ann. Inst. Océanogr. 59(1): 37-56

Hily, C. (1984). Variabilité de la macrofaune benthique dans les milieux hypertrophiques de la rade de Brest. Thèse de Doc.ès Sciences, Univ. Bretagne Occidentale, Brest

Hily, C. (1989). La mégafaune des substrat meubles de la rade de Brest: Pré-échantillonnage par video sous-marine. Cah. Biol. Mar. 30: 433-454

Hily, C., Le Foll, D. (1990). Distribution des supports coquilliers sur les fonds meubles infralittoraux: rôle des perturbations physiques et conséquences sur l'abondance et la distribution d'une population de Chlamys varia, ser. III. C.R. Acad. Sci., Paris, p. 187-192

Hily, C. (1991). Is the activity of benthic suspension feeders a factor controlling water quality in the Bay of Brest? Mar Ecol. Prog. Ser. 45: 179-188

Hutchinson, G. E. (1957). A treatise on Limnology. Wiley Press, New York

Kerambrun, L. (1984). Contribution à l'étude de la fertilité des fonds rocheux côtiers de Bretagne. Thèse de $3^{\text {eme }}$ cycle. Univ. Bretagne Occidentale, Brest

Kjellman, F. R. (1878). Über Algenregionen und Algenformation im ostlichen Skagerrak. Bihang. K. Sv. Vet. Akad. Hands. Bd. 5, VI: 1-35

Lebart, L., Morineau, A., Fenelon, J. P. (1982). Traitement des données statistiques. Dunot Ed., Paris

Legendre, L., Legendre, P. (1979). Ecologie numérique. Le traitement multiple des données écologiques. Masson Ed., Paris

Lieberman, M. J., David, M. J., Lieberman, D. (1979). Ecology of subtidal algae on seasonally devastated cobble substrates off Ghana. Ecology 60: 1151-1161

Lieberman, M., David, M. J., Lieberman, D. (1984). Factors influencing algal species assemblages on reef and cobble substrata off Ghana. J. exp. mar. Biol. Ecol. 75: 129-143

Lubchenco, J., Menge, B. A. (1978). Community development and persistence in a low rocky intertidal zone. Ecol. Monogr, 48: 67-94

Ojeda, F. P., Dearborn, J. H. (1989). Community structure of macroinvertebrates inhabiting the rocky subtidal zone in 
the Gulf of Maine: seasonal and bathymetric distribution. Mar. Ecol. Prog. Ser, 57: 147-161

Paine, R. T. (1966). Food web complexity and species diversity. Am. Nat. 100: 65-75

Parke, M. C., Dixon, P. S. (1976). Check list of British marine algae. Third revision. J. mar. biol. Ass. U.K. 56: 527-594

Potin, P., Floc'h, J. Y., Augris, C., Cabioch, J. (1990). Annual growth rate of the calcareous red alga Lithothamnion corralioides (Corraliales, Rhodophyta) in the Bay of Brest. Hydrobiologia 204/205: 263-267

Salomon, J. C. (1980). Sur un procédé numérique d'exploitation de données courantométriques (rapp. Int. CNRS). In S.A.U.M. (Schéma d'Aménagement et d'Utilisation de la Mer) de la rade de Brest. Ministère de l'Environnement/Ministère des Transports/D.D.E., Brest, p. 34

Sears, J. R., Wilce, R. T. (1975). Sublittoral, benthic marine algae of southern Cape Cod and adjacent islands: seasonal periodicity, associations, diversity and floristic composition. Ecol. Monogr. 45: 337-365

This article was submitted to the editor
Sousa, W. P. (1979). Disturbance in marine intertidal boulder fields: the nonequilibrium maintenance of species diversity. Ecology 60(6): 1225-1239

Sprugel, D. G. (1976). Dynamic structure of wave generated Abies balsamea forests in the northeastern United States. J. Ecology 64: 1225-1239

Sutherland, J. P. (1981). The fouling community at Beaufort, North Carolina: a study in stability. Am. Nat. 108: $859-873$

Treguer, P., Queguiner, B. (1989). Seasonal variations in conservative and non-conservative mixing of nitrogen compounds in a west European macrotidal estuary. Oceanologica acta 12(4): $371-380$

Underwood, A. J., Kennelly, S. J. (1990). Ecology of marine algae on rocky shores and subtidal reefs in temperate Australia. Hydrobiologia 192: 3-20

Waern, M. (1958). Phycological investigations of the Swedish west coast. 1. Introduction and study of the Gaso, shell bottom. Svensk Bot. Tidsk. 52: 319-342

Manuscript first received: April 15, 1991 Revised version accepted: June 18, 1992 